\title{
RMetS
}

Royal Meteorological Society

\section{Assimilation of Radial Velocity and Reflectivity Data from Coastal WSR-88D Radars using an Ensemble Kalman Filter for the Analysis and Forecast of Landfalling Hurricane Ike (2008)}

\author{
Jili Dong and Ming Xue \\ Center for Analysis and Prediction of Storms and School of Meteorology, University of Oklahoma, Norman, OK, USA \\ ${ }^{*}$ Correspondence to: M. Xue, Center for Analysis and Prediction of Storms, National Weather Center, Suite 2500, 120 \\ David L. Boren Blvd, Norman, OK 73072, USA. E-mail: mxue@ou.edu
}

\begin{abstract}
Ensemble Kalman filter (EnKF) assimilation and forecasting experiments are performed for the case of Hurricane Ike (2008), the third most destructive hurricane hitting the USA. Data from two coastal WSR-88D radars are carefully quality controlled before assimilation. In the control assimilation experiment, reflectivity $(Z)$ and radial velocity $\left(V_{\mathrm{r}}\right)$ data from two radars are assimilated at $10 \mathrm{~min}$ intervals over a $2 \mathrm{~h}$ period shortly before Ike made landfall. A 32-member forecast ensemble is initialized by introducing both mesoscale and convective-scale perturbations to the initial National Centers for Environmental Prediction (NCEP) operational global forecast system (GFS) analysis background, and the ensemble spread during the analysis cycles is maintained using multiplicative covariance inflation and posterior additive perturbations. The radar data assimilation results in much improved vortex intensity and structure analysis over the corresponding GFS analysis. Compared with the forecast starting from the GFS analysis, the forecast intensity, track and structure of Ike over a $12 \mathrm{~h}$ period are much improved in both deterministic and ensemble forecasts. Assimilation of either $V_{\mathrm{r}}$ or $Z$ leads to improvement in the forecasts, with $V_{\mathrm{r}}$ data exhibiting much greater impacts than $Z$ data. With the $2 \mathrm{~h}$ assimilation window, $30 \mathrm{~min}$ assimilation intervals produced results similar to 10 min intervals, while 60 min intervals were found to be too long. The ensemble forecasts starting from the EnKF analyses are found to be mostly better than the corresponding deterministic forecast, especially after ensemble post-processing, such as probability matching for precipitation. Precipitation equitable threat scores were calculated and compared. Copyright (c) 2012 Royal Meteorological Society
\end{abstract}

Key Words: hurricane forecast; radar data assimilation

Received 27 August 2011; Revised 23 February 2012; Accepted 16 April 2012; Published online in Wiley Online Library

Citation: Dong J, Xue M. 2012. Assimilation of Radial Velocity and Reflectivity Data from Coastal WSR-88D Radars using an Ensemble Kalman Filter for the Analysis and Forecast of Landfalling Hurricane Ike (2008). Q. J. R. Meteorol. Soc. DOI:10.1002/qj.1970

1. Introduction

Landfalling hurricanes (and tropical cyclones in general) can pose deadly threats to many lives and cause billions of dollars of damage. Accurate prediction of hurricane track, intensity and associated rainfall can save many lives and properties. The official forecasts of hurricane track from the US National Hurricane Center (NHC hereafter) have improved greatly in recent years (Rappaport et al., 2009; Cangialosi and Franklin, 2011), but improvement in hurricane intensity forecasting 
has been limited (Houze et al., 2007). The accuracy of the current operational hurricane intensity forecasts is more or less at the same level as in the 1990s (Rappaport et al., 2009).

Many efforts have been made to identify the factors affecting intensity prediction (Anthes, 1982; Emanuel, 2005; Krishnamurti et al., 2005; Rogers et al., 2006; Li and Pu, 2009; Khain et al., 2010). These factors include surface heat and moisture fluxes, sea-surface temperature and environmental wind shear. Internally, intensity forecasting is believed to be closely related to mesoscale and small-scale wind, cloud and precipitation structures (Houze et al., 2007; Wang, 2009). While hurricane track is primarily determined by large-scale steering flow, some of the vortex-scale or even smaller-scale features and effects, such as the beta drift effect, convection distribution, vertical structure, microphysics processes and vortex asymmetries can also influence track forecasting (Chan and Williams, 1987; Fiorino and Elsberry, 1989; Flatau et al., 1994; Davis and Bosart, 2002; Fovell et al., 2009, 2010). Radar is one of the most effective observation platforms for providing information on hurricane structures at high temporal and spatial resolutions. Radial velocity and reflectivity observed by Doppler weather radars can provide important information on the wind and microphysics properties of hurricanes at the vortex and convective rainband scale (Marks, 2003; Weng et al., 2011; Zhang, 2011; Zhang et al., 2011).

High-resolution numerical models have proven to be a very promising tool for hurricane track and intensity forecasting (Chen et al., 2007; Davis et al., 2008). To improve the ability of numerical models in predicting hurricanes, some studies have examined the sensitivity of forecast to model resolution and physics parametrizations (McFarquhar et al., 2006; Fierro et al., 2009), while others have focused on improving the initial conditions through data assimilation (DA). Assimilating bogus vortex data has resulted in improved hurricane forecasts (Zou and Xiao, 2000; Pu and Braun, 2001; Xiao et al., 2009a; Kwon and Cheong, 2010; Zou et al., 2010). Without resorting to vortex bogusing, radar observations have been used to define vortex initial conditions, leading to improved forecasts in both track and intensity when using high-resolution models (e.g. Xiao et al., 2007; Zhao and Jin, 2008; Pu et al., 2009; Xiao et al., 2009b; Zhang et al., 2009; Zhao and Xue, 2009; Zhao et al., 2012a, 2012b).

All DA studies cited above used a three-dimensional variational (3D-Var) DA method, except for Zhang et al. (2009). Using static background error statistics that usually do not have any knowledge about the presence of a hurricane, $3 \mathrm{D}-$ Var is not the most effective method for localized, rapidly evolving hurricanes. The lack of flow-dependent cross-variable covariance also prevents effective extraction of information contained in remotely sensed radar data that measure only radial velocity and reflectivity. With such limitations, 3D-Var is not very effective at, or even capable of, directly assimilating reflectivity data, especially when using a mix-phase microphysics scheme. Among more advanced DA methods, the ensemble Kalman filter (EnKF) employs ensemble forecasts to estimate flow-dependent background error covariance, including cross-covariances among variables, and thus has advantages over 3D-Var.

The EnKF was first proposed by Evensen (1994) and has since seen widespread applications in geophysical science as well as other fields. Its relatively simple formulation and easy implementation compared with the four-dimensional variational (4D-Var) DA method allow it to enjoy great popularity within the research community. The EnKF has found its way into operational NWP systems, including creating initial conditions for ensemble forecasting (e.g. Houtekamer et al., 2009). It has also been shown to improve tropical cyclone track forecasting within a global forecasting model (Hamill et al., 2011) and can be used for hurricane predictability research also (e.g. Sippel and Zhang, 2010; Zhang, 2011).

Previous studies have demonstrated that EnKF is a powerful tool for assimilating radar data, including radial velocity and reflectivity for convective thunderstorms (Dowell et al., 2004; Zhang et al., 2004; Tong and Xue, 2005; Xue et al., 2006; Aksoy et al., 2009). For tropical cyclones, Zhang et al. (2009) assimilated radar radial velocity data from coastal weather radars for hurricane Humberto (2007). Using EnKF, the hurricane's position and intensity were accurately analyzed and deterministic forecasts resulting from the ensemble mean analyses showed improvement in track and intensity over both operational forecasts and forecasts initialized with a 3D-Var scheme. Most recently, Li et al. ( 2012) applied an ensemble-3D-Var hybrid method to assimilate radial velocity data from two coastal radars, and demonstrated the advantage of using ensemble-derived flow-dependent covariance over static 3D-Var covariance for hurricane initialization.

The assimilation of reflectivity data has produced positive impacts on hurricane forecasts within 3D-Var frameworks (Xiao et al., 2007; Zhao and Jin, 2008; Zhao and Xue, 2009), but so far reflectivity data have not been assimilated using EnKF for tropical storms according to our knowledge. Even within 3D-Var frameworks, reflectivity has been assimilated by assuming a warm-rain process only (Xiao et al., 2007), or utilizing a post-3D-Var adjustment procedure (Zhao and Jin, 2008), or using a separate complex cloud analysis package (Zhao and Xue, 2009); these practices indicate difficulties with direct assimilation of reflectivity data using 3D-Var. We note here that even for continental convection where most of the EnKF radar data assimilation research has occurred, the assimilation of reflectivity data using EnKF is also still an active topic for research (e.g. Dowell et al., 2011). There are more uncertainties with the reflectivity observation operators due to the involvement of microphysics size distributions, etc., and the nonlinearity of the operators poses additional challenges (Jung et al., 2008, 2010). Effective assimilation of reflectivity data usually requires reliable cross-variable covariances among state variables, including those of microphysical species while microphysics parametrization errors may significantly affect the reliability of covariance and filter performance (Snook et al., 2011; Jung et al., 2012).

This study attempts to investigate for the first time the assimilation of radar radial velocity and reflectivity observations with EnKF for a hurricane, either at the same time or individually. One of the variants of EnKF, the serial ensemble square root filter (EnSRF; Whitaker and Hamill, 2002) that has been used extensively for thunderstorm radar assimilation, is used in this study. Extending previous research, this study also investigates the impact of EnKF assimilation of radar data on quantitative precipitation forecasting (QPF) for a hurricane.

The rest of this article is organized as follows. Section 2 briefly introduces the Hurricane Ike case, the forecast model, radar observations, EnKF algorithm and experiment 


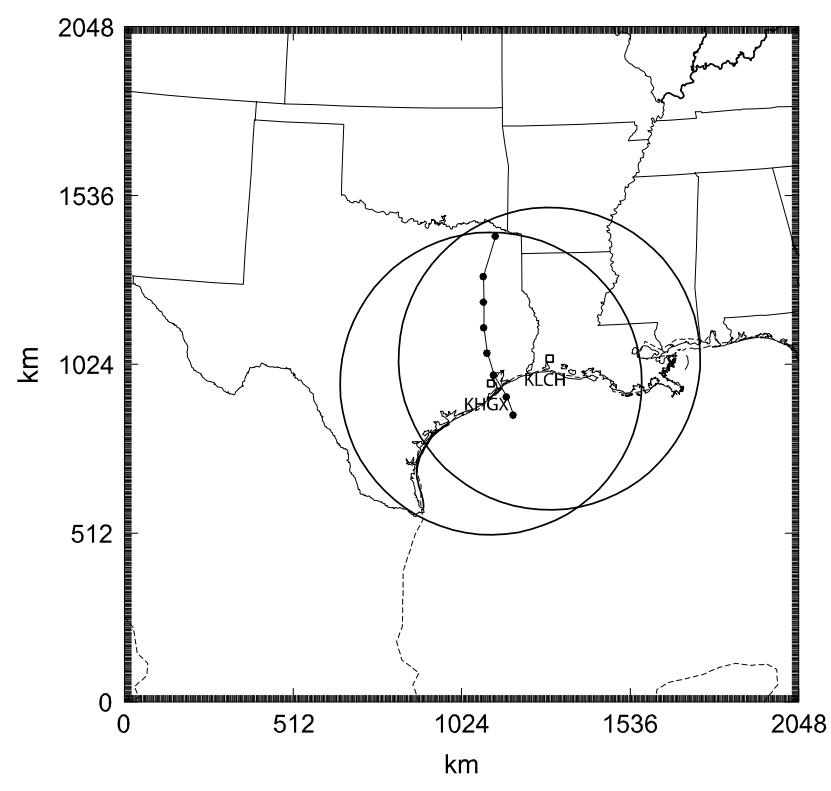

Figure 1. The physical domain of the numerical simulation, the best track (denoted by black dots) and radar coverage for Ike. The position of two radars are denoted by rectangles. The range circles of the Houston-Galveston, Texas (KHGX) and Lake Charles, Louisiana (KLCH) radars are for a maximum of $460 \mathrm{~km}$. The best track starts from 0300 UTC September 13 to 0000 UTC September 14, plotted every $3 \mathrm{~h}$.

set-up. Ensemble spreads and innovation statistics during the analysis cycles are discussed in section 3. Section 4 discusses the impact of radar data on the deterministic forecasts of intensity, track and precipitation while ensemble forecasting results are shown in section 5 . Section 6 examines the sensitivity to DA intervals and the use of single radars. A summary is given in section 7 .

\section{Hurricane Ike (2008), prediction model, observations and data assimilation experiment setting}

\subsection{Hurricane Ike (2008)}

Hurricane Ike (2008) was the most intense hurricane during the 2008 Atlantic hurricane season, reaching category 4 over the western Atlantic before making landfall over Cuba at category 3. It made landfall along the US coast near Galveston, Texas at 0700 UTC 13 September as a category 2 hurricane. After the first landfall over Galveston, Ike passed over San Leon, Texas and made its final landfall near Baytown, Texas around 0900 UTC. At and after landfall, Ike took a northwest, north, then northeast path (Figure 1) and weakened to a tropical storm around 1800 UTC September 13 in eastern Texas. Over its inland path, Ike brought heavy precipitation and damages to 11 US states, making it the third costliest hurricane in US history, only after Hurricane Katrina (2005) and Hurricane Andrew (1992). This study investigates the impact of assimilating radar reflectivity and radial velocity data from two coastal WSR-88D radars using EnKF.

\subsection{The prediction model}

The advanced regional prediction system (ARPS; Xue et al., $2000,2001,2003$ ) is used in this study as the prediction model. The prognostic state variables associated with the ARPS options used include velocity components $u, v$ and $w$, potential temperature $\theta$, pressure $p$, turbulence kinetic energy (TKE), water vapour mixing ratio $q_{\mathrm{v}}$, and the mixing ratios of cloud water, rainwater, ice, snow and hail $\left(q_{\mathrm{c}}, q_{\mathrm{r}}, q_{\mathrm{i}}, q_{\mathrm{s}}\right.$ and $q_{\mathrm{h}}$, respectively). A $515 \times 515 \times 53$ grid with a horizontal resolution of $4 \mathrm{~km}$ defines the whole physical domain of $2048 \times 2048 \times 25 \mathrm{~km}^{3}$ (Figure 1). Mean vertical grid spacing is $500 \mathrm{~m}$, with a vertical grid stretching scheme that has a grid spacing of $50 \mathrm{~m}$ at the surface, yielding 14 model levels within the lowest $1 \mathrm{~km}$. The lowest model level for scalar variables and horizontal wind components is at $25 \mathrm{~m}$ above surface. The Lin et al. (1983) three-ice microphysical scheme is used. Subgrid-scale turbulence mixing is handled by the 1.5-order TKE-based turbulence parametrization after Deardorff (1980). while within the convective planetary boundary layer (PBL) a non-local vertical mixing length is used based on Sun and Chang (1986). The PBL scheme was tested initially in Xue et al. (1996) for ARPS. The surface fluxes are parametrized using stability and roughness-lengthdependent formulations based on Businger et al. (1971) and Deardorff (1972), and over the ocean the roughness length is wind-speed dependent (Donelan et al., 1993). An alternative formulation for the drag coefficient for highwind conditions based on more recent observational studies (Powell et al., 2003; Donelan et al., 2004) was also tested but the results were found to be insensitive to the particular formulation for the case.

Other physics parametrizations include the National Aeronautics and Space Administration (NASA) Goddard Space Flight Center (GSFC) long- and short-wave radiation parametrizations, a two-layer force-restore soil-vegetation model (Ren and Xue, 2004) initialized with the National Centers for Environmental Prediction (NCEP) global forecast system (GFS) land-surface model state. Fourthorder momentum advection is used in both the horizontal and vertical. A second-order monotonic flux-corrected transport (FCT) scheme (Zalesak, 1979) is applied to potential temperature, water variables and TKE. Details on these physics and computational options can be found in Xue et al. (1995) and Xue et al. (2001, 2003).

\subsection{Radar observations, quality control and observation operators}

Coastal WSR-88D radars at Houston-Galveston, Texas (KHGX) and Lake Charles, Louisiana $(\mathrm{KLCH})$ provided the coverage for Ike when it approached the Texas coast (Figure 1). Reflectivity $(Z)$ and radial velocity $\left(V_{\mathrm{r}}\right)$ data from these two radars are assimilated with observation error standard deviations specified as $2 \mathrm{dBZ}$ and $1 \mathrm{~m} \mathrm{~s}^{-1}$, respectively. For the quality control, level-II $Z$ data are processed with the $88 \mathrm{~d} 2$ arps program in the ARPS system (Brewster et al., 2005) to remove ground clutter, speckles and other artefacts. The level-II $\mathrm{Vr}$ data are manually quality-controlled for each elevation using interactive SOLO software (Oye et al., 1995) available from the National Center for Atmospheric Research (NCAR) to ensure correct velocity de-aliasing.

After quality control, the radar data on the scan elevations are bilinearly interpolated to the ARPS scalar variable column locations but kept on the radar elevation levels. Since the range resolution of the level-II data $(250 \mathrm{~m}$ for radial velocity and $1 \mathrm{~km}$ for reflectivity) is much higher than the model grid spacing of $4 \mathrm{~km}$, only the data surrounding the 
model columns are used in the interpolation; this procedure represents a form of data thinning. During the assimilation period, volume coverage patterns (VCP) modes 12 and 121 were employed by KHGX and KLCH, respectively, providing a dense vertical coverage for lower elevation angles. No vertical interpolations are performed for the observations, i.e. the data are kept on the elevation levels and assimilated directly. All data within a 5 min scan volume closest to the analysis time are assumed to be valid at the analysis time. Negative reflectivity values are set to zero and still assimilated, mainly for the purpose of suppressing possible spurious convection. The radial velocity data are assimilated only where observed $Z$ is larger than $10 \mathrm{dBZ}$. These are consistent with recommendations of Tong and Xue (2005).

The forward observation operator for $V_{\mathrm{r}}$ data is based on the formula of Tong and Xue (2008), that includes the hydrometeor terminal velocities. The reflectivity formula of Tong and Xue (2008) is used, which calculates the model version of reflectivity in $\mathrm{dBZ}$ from the mixing ratios of rain, snow and hail. A transition zone from dry to wet hail is defined in the $-2.5^{\circ}$ to $2.5^{\circ} \mathrm{C}$ temperature layer. Because the observations are at the radar elevations, a vertical beam pattern weighting function using a Gaussian power-gain function is used to bring the grid point values of radial velocity and reflectivity to the elevation levels, as described in Xue et al. (2006). This weighting function allows the data on the elevation levels to directly impact grid levels within the radar beam width, while other levels within the vertical localization radius can be updated by the filter through spatial covariances.

\subsection{EnKF experiments and settings}

A baseline control forecast without radar DA (CNTL) is run from 0600 UTC 13 September, initialized with NCEP GFS analysis valid at the same time (Table 1). In all other experiments with EnKF radar DA, the forecast ensemble is created by adding mesoscale and convective perturbations in two steps. In the first step, a single $4 \mathrm{~h}$ forecast is run from 1800 UTC September 12 GFS analysis interpolated to the $4 \mathrm{~km}$ model grid, and mesoscale perturbations are added to the forecast at 2200 UTC in the entire model domain to create an initial ensemble of 32 members. These perturbations are created by smoothing Gaussian random perturbations with zero mean using a two-dimensional recursive filter (Purser et al., 2003), with a horizontal decorrelation scale of $100 \mathrm{~km}$ (see, e.g. Huang, 2000; Jung et al., 2012). The perturbations are scaled to have standard deviations of $2 \mathrm{~m} \mathrm{~s}^{-1}$ for $u$ and $v, 1 \mathrm{~K}$ for $\theta$ and $1 \mathrm{hPa}$ for $p$. For $q_{\mathrm{v}}$, the relative standard derivation is $10 \%$ of the unperturbed value, to avoid excessively large absolute perturbations at the upper levels. Other state variables are not perturbed in this step. Six-hour-long ensemble forecasts are first carried out from these perturbed initial conditions to develop evolved background error covariance, which hopefully samples the mesoscale environmental uncertainties.

In the second step, at 0400 UTC 13 September, another set of convective-scale perturbations with a smaller horizontal decorrelation scale of $12 \mathrm{~km}$ and a vertical decorrelation scale of $4 \mathrm{~km}$ is added to the ensemble forecast fields, but only in regions where observed $Z$ exceeds $10 \mathrm{dBZ}$. These perturbations are created by applying a fifth-ordercorrelation smoothing function after Tong and Xue (2008). Their standard deviations are $2 \mathrm{~m} \mathrm{~s}^{-1}$ for the wind components, $2 \mathrm{~K}$ for $\theta, 10 \%$ for $q_{\mathrm{v}}$, and $1 \mathrm{~g} \mathrm{~kg}^{-1}$ for all microphysical variables. The above spatial scales and magnitudes of perturbations were found to yield the best results through many assimilation experiments.

The lateral boundary conditions are from the $6 \mathrm{~h}$ operational GFS analyses and $3 \mathrm{~h}$ forecasts in between, available on the $0.5^{\circ}$ grid. They are also perturbed by adding mesoscale perturbations. Within ARPS, linear time interpolations are performed between the boundary condition times.

Our first set of three DA experiments, ExpVr, ExpZ and ExpAll (also called Exp10Min), employs 10 min assimilation cycles. They assimilate $V_{\mathrm{r}}$ alone, $Z$ alone and both $V_{\mathrm{r}}$ and $Z$, respectively (Table 1). In these experiments, the first EnKF analysis of radar data occurs at 0410 UTC, $10 \mathrm{~min}$ after the convective-scale perturbations are introduced into the forecast ensemble, and the assimilation cycles end at 0600 UTC 13 September, around $1 \mathrm{~h}$ before the landfall. Two additional experiments, named Exp30Min and Exp60Min (Table 1), are the same as ExpAll but assimilate the data every 30 and $60 \mathrm{~min}$, respectively. In two more experiments, ExpKHGX and ExpKLCH, instead of using data from both radars, a single radar is used: $\mathrm{KHGX}$ or $\mathrm{KLCH}$, respectively. At the end of the assimilation window, at 0600 UTC, an $18 \mathrm{~h}$ long deterministic forecast and an ensemble of forecasts are run until 0000 UTC 14 September. The DA experiments and no-radar control experiment CNTL are illustrated in Figure 2.

To reduce sampling error caused by the small ensemble and to account for inevitable model error, prior multiplicative covariance inflation of 5\% (Tong and Xue, 2005; Xue et al., 2006) before each analysis and posterior additive inflation after each analysis are applied at the model points within the influence range of available radar observations. The multiplicative inflation is applied to all 11 state variables. The additive noise has a Gaussian distribution with zero mean and the standard deviations are $1 \mathrm{~m} \mathrm{~s}^{-1}$ for $u, v$ and $w$, and $1 \mathrm{~K}$ for $\theta$. The additive noise has a horizontal decorrelation scale of $9 \mathrm{~km}$ and a vertical scale of $4 \mathrm{~km}$, achieved by applying the fifth-order filters (Tong and Xue, 2008). Whitaker and Hamill (2010) found that the best result is obtained with the combination of multiplicative and 'evolved' additive inflations in their global model study, while Dowell and Wicker (2009) documented benefit of additive noise for thunderstorm analysis. For all of our experiments, a covariance localization to alleviate the negative impacts of noisy remote covariance has cut off radii of $12 \mathrm{~km}$ in the horizontal and $4 \mathrm{~km}$ in the vertical. The inflation and localization parameters were chosen based on a number of sensitivity experiments; so were the magnitudes of the initial and additive perturbations.

In our earlier sensitivity experiments, it was found that using reflectivity to update the wind, potential temperature and water vapour mixing ratio had a negative impact on the hurricane intensity analysis. Hence, in our EnKF algorithm, the reflectivity data are used to update only pressure and microphysical variables, $q_{\mathrm{c}}, q_{\mathrm{i}}, q_{\mathrm{r}}, q_{\mathrm{s}}$ and $q_{\mathrm{h}}$. The radial velocity data update all of the 11 state variables, i.e. $u, v$, and $w, \theta, p, q_{\mathrm{v}}$, and all microphysical variables. The updating of non-velocity variables is achieved through the flowdependent cross-covariance derived from the ensemble. 
Table 1. Summary of experiments with no radar data assimilation, and with radar data assimilation using different observation types, assimilation intervals or the number of radars.

\begin{tabular}{lccc}
\hline Experiment & Observations assimilated & Radar assimilation interval (min) & Single or both radars \\
\hline CNTL & No radar DA & NA & NA \\
ExpVr & $V_{\mathrm{r}}$ & 10 & Both \\
ExpZ & $Z$ & 10 & Both \\
ExpAll (Exp10Min) & $V_{\mathrm{r}}+Z$ & 30 & Both \\
Exp30Min & $V_{\mathrm{r}}+Z$ & 60 & Both \\
Exp60Min & $V_{\mathrm{r}}+Z$ & 10 & Both \\
ExpKHGX & $V_{\mathrm{r}}+Z$ & 10 & KHGX only \\
ExpKLCH & $V_{\mathrm{r}}+Z$ & KLCH only \\
\hline
\end{tabular}

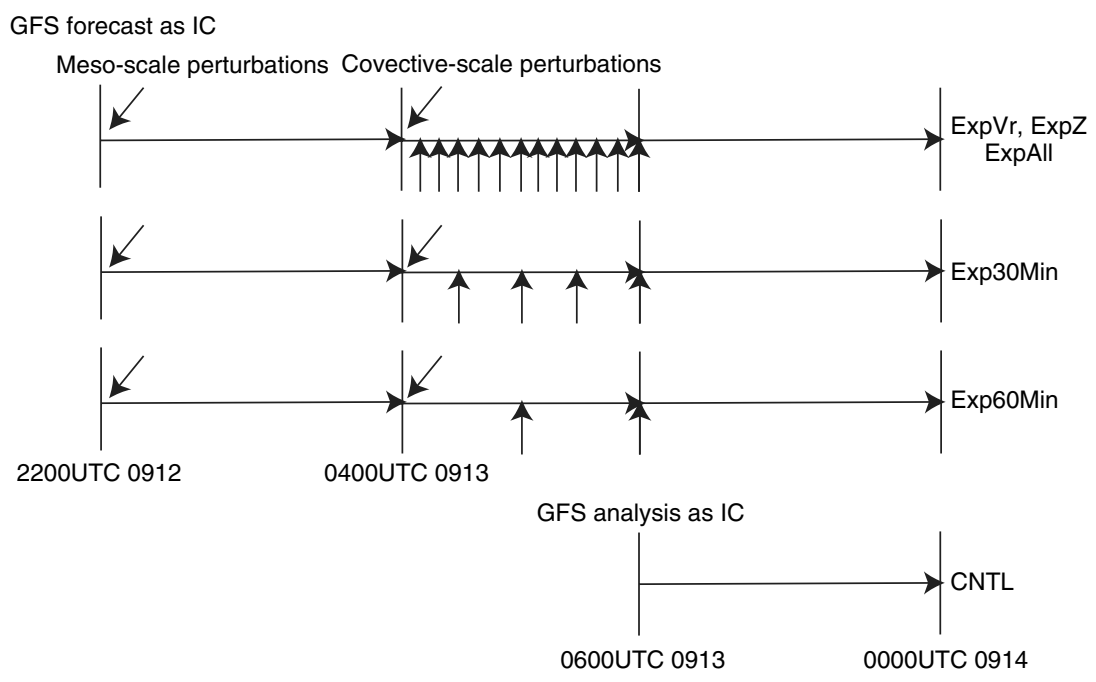

Figure 2. The data assimilation and control simulation (CNTL) schemes. From the top: 10 min assimilation interval, 30 min assimilation interval, 60 min assimilation interval and CNTL simulation. The vertical arrows denote the assimilation of radar observations. The tilted arrows denote the addition of perturbations to generate the initial ensemble members.

\section{Ensemble spread, observation innovation statistics and analysis increments}

The EnKF relies on a sufficiently accurate estimate of the background error covariance to update the state variables in a dynamically consistent way. Due to sampling error and the lack of explicit representation of model errors, the forecast ensemble tends to be underdispersive. Maintaining adequate ensemble spread is necessary to prevent filter divergence. An examination of the spread of the state variables in the precipitation region during the analysis cycles of ExpAll (Figure 3) reveals that spread reductions occur with each EnKF analysis for all state variables (even for pressure that is not directly observed), with larger reductions during the first few cycles and stabilizing in later cycles. Despite the gradual reduction of the forecast spread in the subsequent cycles, the standard deviations from the ensemble members are maintained at levels between 2.5 and $3 \mathrm{~m} \mathrm{~s}^{-1}$ for $u$ and $v$, between 0.06 and $0.14 \mathrm{~g} \mathrm{~kg}^{-1}$ for $q_{\mathrm{c}}$, and between 0.3 and $0.35 \mathrm{hPa}$ for pressure. These errors are at a level expected of good quality analyses, and their maintenance was helped by the multiplicative and additive inflations applied.

The observation innovations, or the r.m.s. differences between the observations and the model states in the observation space, measure how well the model state fits the observations. It is typically denoted as $y-\mathrm{H}(x)$ where $y$ is the observation vector, $x$ the state vector and $H$ the observation operator. The r.m.s. innovations of ExpVr, ExpZ and ExpAll averaged in the precipitation region are shown in Figure 4 for the analyses and background forecasts. With respect to both radars, the r.m.s. innovations of $V_{\mathrm{r}}$ have the largest reduction in the first two assimilation cycles in both ExpVr and ExpAll. In these two experiments, after 10 to $20 \mathrm{~min}$ of forecast and analysis, the innovation reductions remain and continue until the end of the analysis cycles. At the end, the r.m.s. innovations of $V_{\mathrm{r}}$ are 2 to $6 \mathrm{~m} \mathrm{~s}^{-1}$, which are much smaller than the initial values of about $10 \mathrm{~m} \mathrm{~s}^{-1}$. This indicates that both the forecast and the analysis states are largely improved by the EnKF DA in terms of their fit to radar observations. In ExpZ, the assimilation of reflectivity does not update model wind fields but only the microphysical variables. The hydrometeor variables are involved in the calculation of terminal velocities and in the $V_{\mathrm{r}}$ observation operator (Tong and Xue, 2008). Through this connection, the assimilation of $Z$ actually slightly improves the $V_{\mathrm{r}}$ innovation against $\mathrm{KLCH}$ data (Figure 4(b)). In ExpAll, the reflectivity analysis improves the microphysics fields and also the covariance structure. Through better state and covariance estimation, $V_{\mathrm{r}}$ in ExpAll is able to improve $V_{\mathrm{r}}$ analysis more than in ExpVr, as shown in Figure 4(a) and Figure 4(b). For the $Z$ innovations in ExpZ and ExpAll, the largest reductions are in the first cycle. At the end, the r.m.s. innovation of $Z$ was around $5 \mathrm{dBZ}$ in those two experiments, much smaller than the initial values of $20 \mathrm{dBZ}$. The assimilation of $V_{\mathrm{r}}$ data has very limited direct impact on the model microphysical fields, as evidenced by the slight innovation increase during the analysis time. Most of the reduction of $Z$ innovation in ExpVr is through the model forecast adjustment during the 

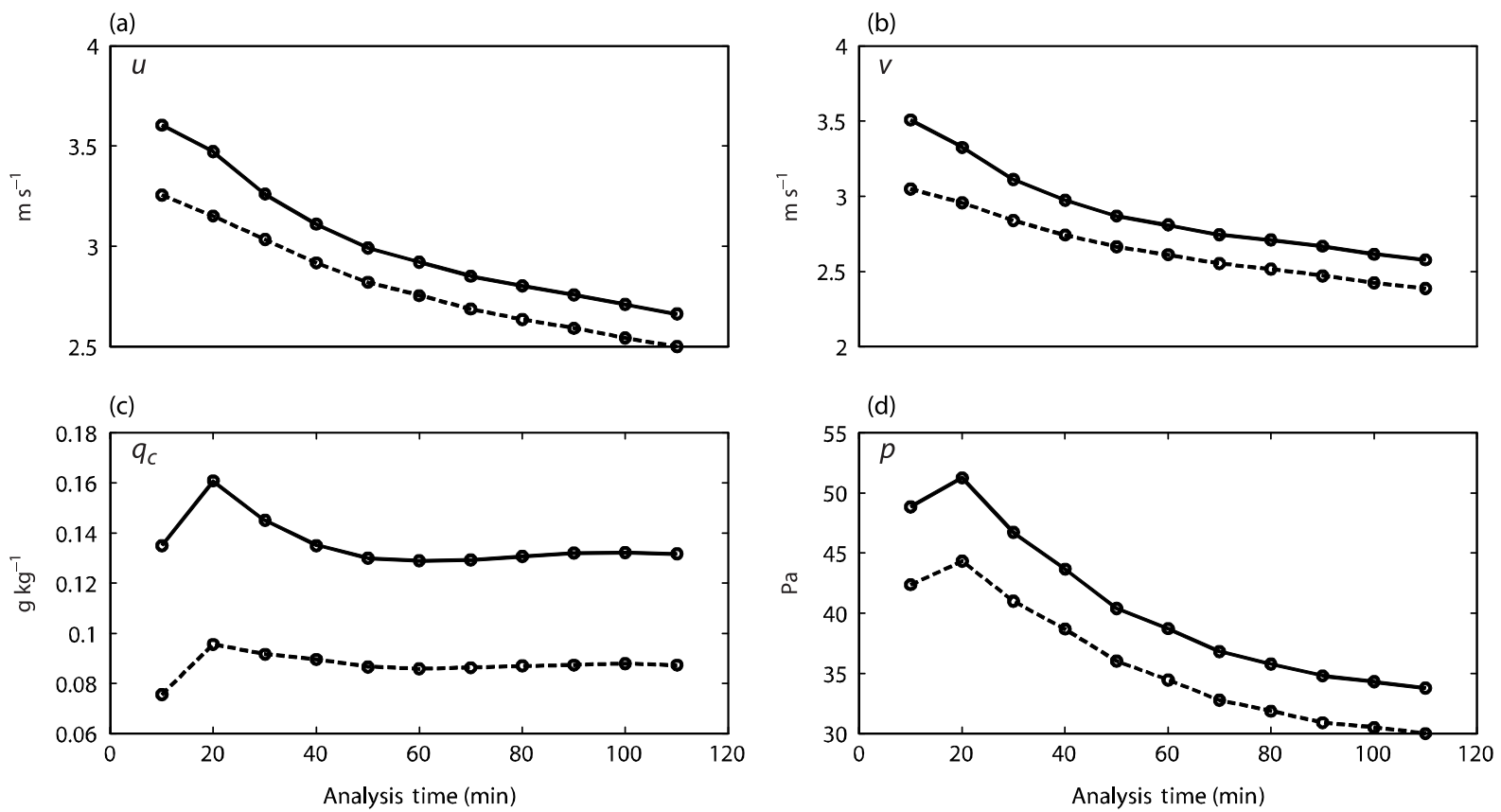

Figure 3. Time evolution of ensemble forecast and analysis spreads, defined as the standard deviations from the ensemble mean, during the EnKF analysis cycles, spatially averaged in precipitation regions where $Z>10 \mathrm{dBZ}$, for (a) $u$, (b) $v,(\mathrm{c})$ cloud water mixing ratio $\left(q_{\mathrm{c}}\right)$, and (d) pressure $(p)$, from experiment ExpAll. Those for the background forecast are depicted by solid lines and those for analysis by dashed lines.

first several cycles, after the wind fields are improved by $V_{\mathrm{r}}$ assimilation.

The analysis and forecast minimum sea-level pressures (MSLPs) during the assimilation cycles are plotted in Figure 5 to further examine the impact on the storm intensity from $V_{\mathrm{r}}$ and $Z$. The best-track MSLPs are also plotted for comparison purposes. The MSLP decreases by about $5 \mathrm{hPa}$ in ExpVr and ExpAll in the first analysis and the storms continue to strengthen over the next $30 \mathrm{~min}$. The reduction of MSLP during the forecast is a result of pressure adjustment to the updated wind fields by $V_{\mathrm{r}}$ data. In the rest of the assimilation cycles, the centre pressures of ExpVr and ExpAll are well maintained at around $955 \mathrm{hPa}$ with little error growth during the forecast. The MSLP of ExpZ changes little during the analysis before 0540 UTC. During the last $20 \mathrm{~min}$, MSLP decreases by $1-3 \mathrm{hPa}$ in the analysis, probably benefiting from a better evolved covariance between the microphysical fields and pressure. The final analyzed centre pressure of ExpZ is much higher than the best track and those of ExpVr and ExpAll.

To better understand the behaviour of the radar data analysis, the increments of horizontal wind components and pressure in the first and last analysis cycles are plotted in Figure 6 and Figure 7 at different model levels for ExpVr, ExpZ and ExpAll. During the first analysis at 0410 UTC, the horizontal wind increments in ExpVr and ExpAll appear to systematically strengthen the hurricane vortex in the background, with the increments showing a wellorganized structure of cyclonic circulation at low to high levels (Figure 6(d), (g), (j), (f), (i) and (l)). At the surface (the first level above ground), the data coverage from the farther located KLCH is small due to the upward tilting of radar beams; this makes the impact come mainly from KHGX and only part of the vortex is strengthened (Figure 6(a) and (c)). Above $3 \mathrm{~km}$, the wind increments decrease with height, probably due to the weaker vortex aloft. The pressure increments in ExpVr and ExpAll are the largest at the lower levels, as are the wind increments; this is consistent with the decreasing vortex intensity with height. The impacts on the pressure from radial winds are determined by the covariance between wind and pressure estimated from the ensemble. For ExpZ, the pressure increments are sporadic and small in the first analysis, indicating weak correlations between the microphysical fields and pressure. Note that the winds are not updated by $Z$ in ExpZ.

At the end of the analysis cycles, the error in the overall vortex of the background forecast has been greatly reduced from the previous cycles and the wind increments are therefore much less organized, indicating that most of the corrections now correspond to the sub-vortex, convective scale structures (Figure 7(a), (d), (g), (j), (c), (f), (i) and (l)). As for ExpZ, there are noticeable low-level pressure increments at the surface, although highly localized. The net effect is the reduction of MSLP (Figure 5).

\section{Data assimilation impact on the analysis and deterministic forecast}

\subsection{Impact on analyzed and forecast hurricane structures}

The fields of wind, pressure and temperature from the GFS analysis and from the final EnKF analyses of ExpZ, ExpVr and ExpAll at 0600 UTC are shown in Figure 8, at the $1 \mathrm{~km}$ height level and in vertical cross-sections through the individual vortex centre of each experiment. With radar data, the centre pressure in the DA experiments is clearly lower than in the GFS analysis (Figure 8(a)-(d)), and the wind speed is larger, especially in ExpVr and ExpAll. The vertical cross-sections show that the maximum wind speed is on the east side of the vortex centre in all cases (Figure 8(e)-(h)). ExpZ has a maximum wind speed of $51 \mathrm{~m} \mathrm{~s}^{-1}$ whereas those of ExpVr and ExpAll are over $59 \mathrm{~m} \mathrm{~s}^{-1}$, compared with the $44 \mathrm{~m} \mathrm{~s}^{-1}$ of GFS analysis. Strong winds exceeding $36 \mathrm{~m} \mathrm{~s}^{-1}$ are confined to the lowest $3 \mathrm{~km}$ in GFS analysis but extend to $7 \mathrm{~km}$ in ExpZ and $10 \mathrm{~km}$ in ExpVr and ExpAll; 
(a)

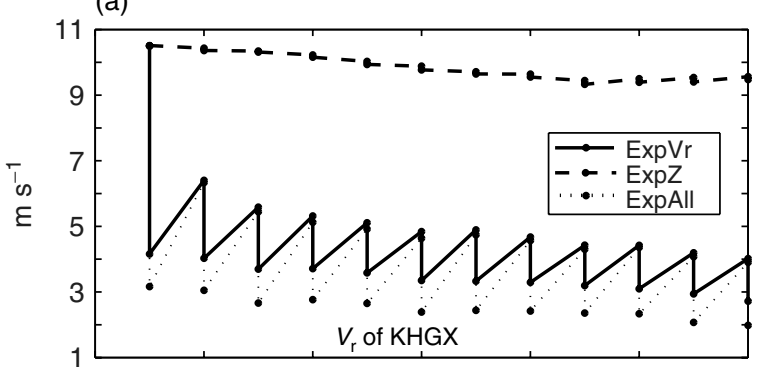

(c)

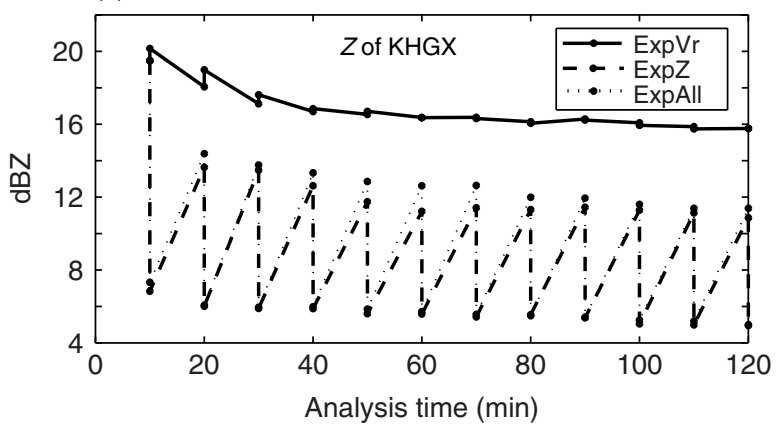

(b)

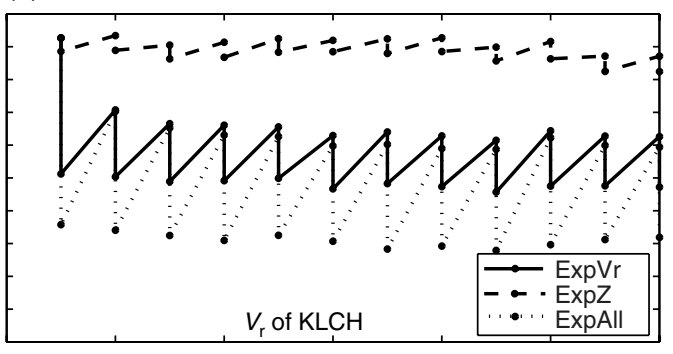

(d)

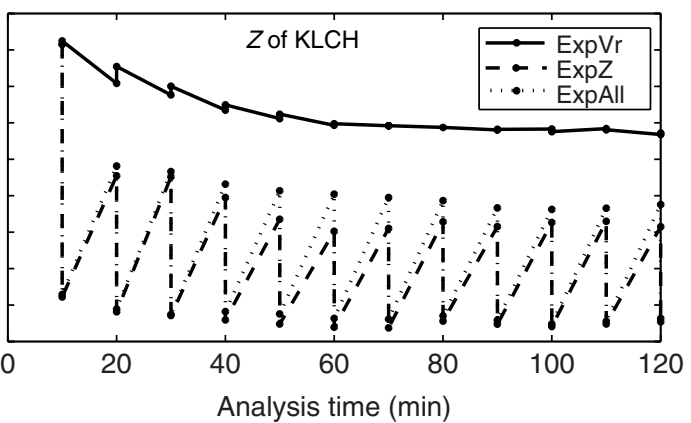

Figure 4. Time evolution of innovation r.m.s. during the analysis cycles, averaged in precipitation regions (observed $Z>10 \mathrm{dBZ}$ ) for (a) $V_{\mathrm{r}}$ of $\mathrm{KHGX}$ and (b) KLCH, (c) Z of KHGX and (d) KLCH from experiments ExpVr (solid), ExpZ (dashed) and ExpAll (dotted).

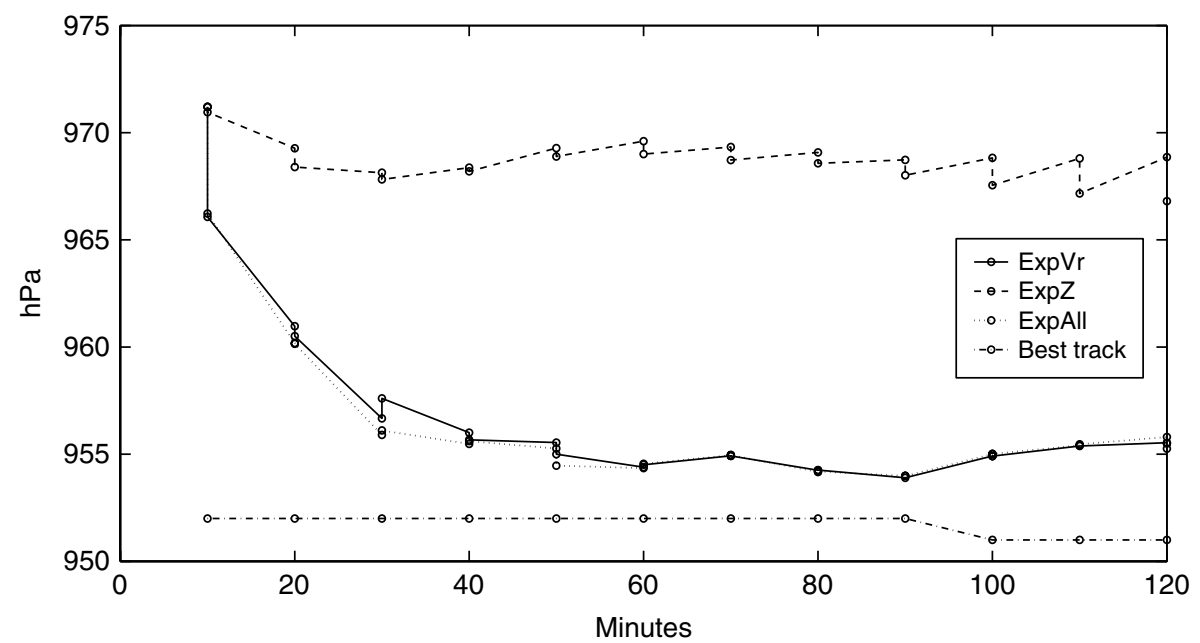

Figure 5. Analysis and forecast minimum sea-level pressures during the analysis cycles in ExpVr, ExpZ and ExpAll. The best-track data are linearly interpolated to $10 \mathrm{~min}$ intervals for comparison.

this indicates that deeper vortices are obtained when radar data are assimilated and $V_{\mathrm{r}}$ data clearly have the most direct impact on the wind fields analyzed.

Figure 8(i)-(l) show the temperature and vertical velocity fields in vertical cross-sections. With the assimilation of reflectivity in ExpZ, a stronger warm core is established at the low to mid-levels (Figure 8(j)) compared with that in GFS analysis (Figure 8(i)), and the warm core is even stronger in ExpVr and ExpAll. Weak updrafts of generally less than $1 \mathrm{~m}$ $\mathrm{s}^{-1}$ are found in ExpZ on both sides of the vortex centre near the radius of maximum wind, while vertical velocity that can be derived from GFS analysis is much weaker (not shown). In ExpVr and ExpAll, the vertical velocity is stronger and has more structures than in ExpZ, apparently due to the additional flow information introduced by the $V_{\mathrm{r}}$ data. A deep updraft of more than $2.2 \mathrm{~m} \mathrm{~s}^{-1}$ is found in ExpAll near $x=1280 \mathrm{~km}$, and such updrafts are usually associated with rain bands (see Figure 7). Clearly, the assimilation of radar observations builds a stronger, tighter and deeper vortex with a warm core that is more consistent with the observed hurricane intensity.

The single east-west cross-section through the vortex centre may not be very representative of the whole vortex. Azimuthally averaged radius-height wind and temperature fields at 0600 UTC are plotted in Figure 9. Compared with CNTL, horizontal winds are stronger in ExpZ with the maximum value exceeding $36 \mathrm{~m} \mathrm{~s}^{-1}$. The maximum winds at low levels are over $48 \mathrm{~m} \mathrm{~s}^{-1}$ in ExpVr and ExpAll. Regions with wind speed exceeding $30 \mathrm{~m} \mathrm{~s}^{-1}$ extend to $10 \mathrm{~km}$ height in ExpVr and ExpAll, deeper than the $9 \mathrm{~km}$ in CNTL and ExpZ. Large horizontal gradients of wind speed are built up around the vortex centre in ExpVr and ExpAll. The regions of strong wind exceeding $36 \mathrm{~m} \mathrm{~s}^{-1}$ extend to $180 \mathrm{~km}$ from the vortex centre in ExpVr and ExpAll. The warm core of ExpZ is slightly deeper than that of CNTL, while ExpVr and ExpAll have even stronger and deeper warm 
(a)

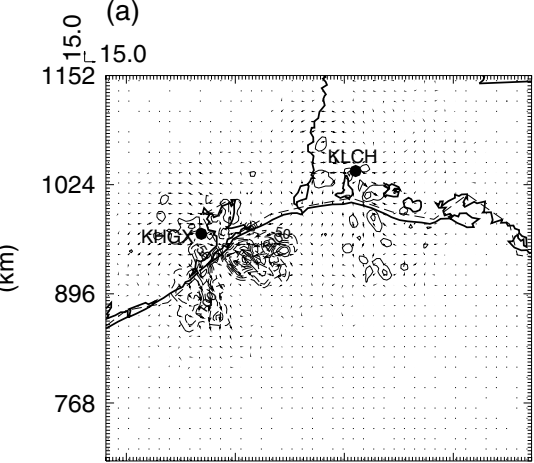

(d)

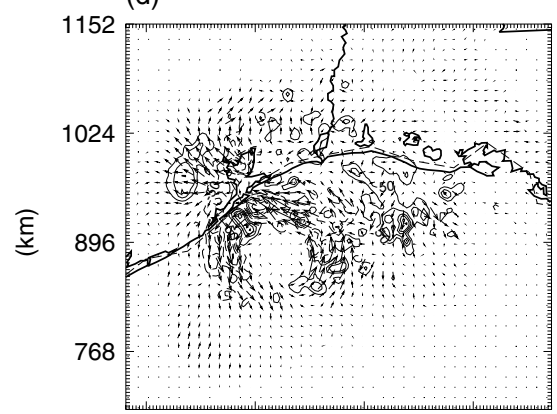

(g)
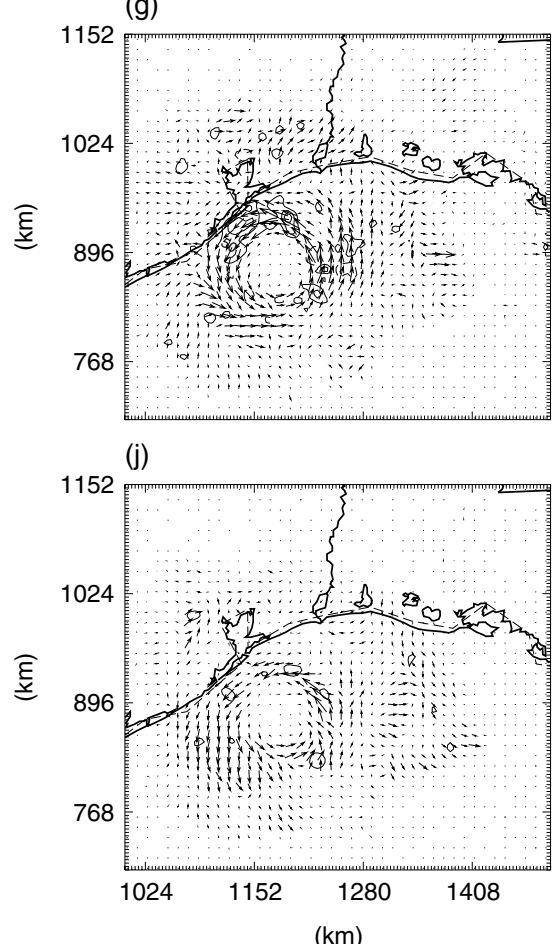

(b)

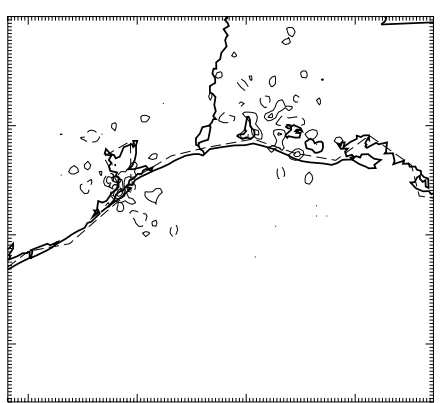

(e)

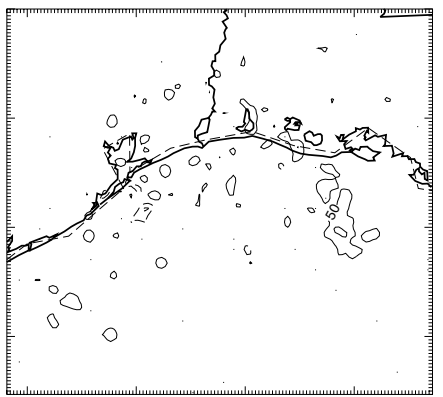

(h)

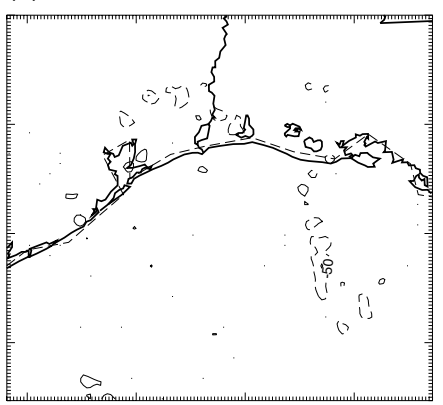

(k)

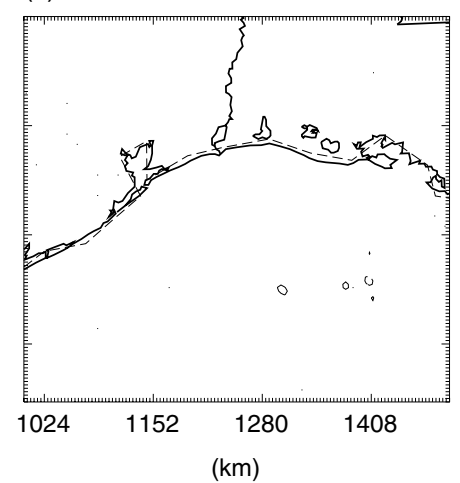

(c)

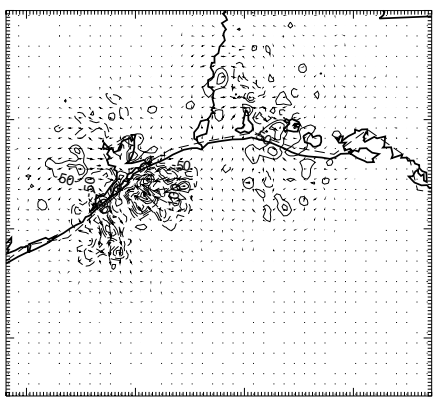

(f)

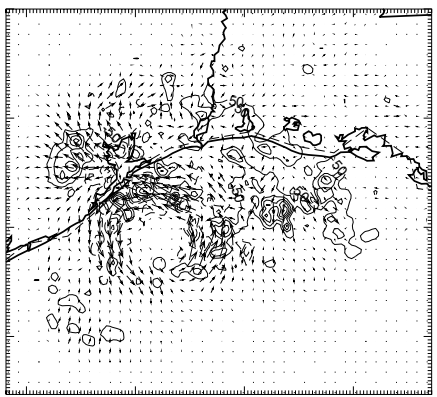

(i)

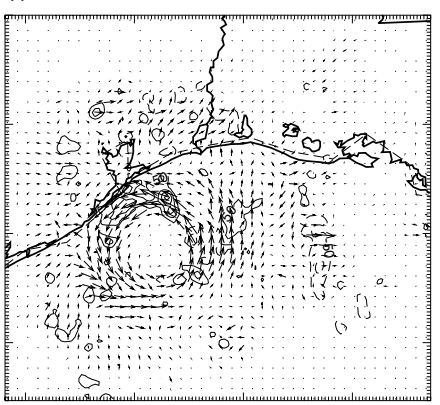

(I)

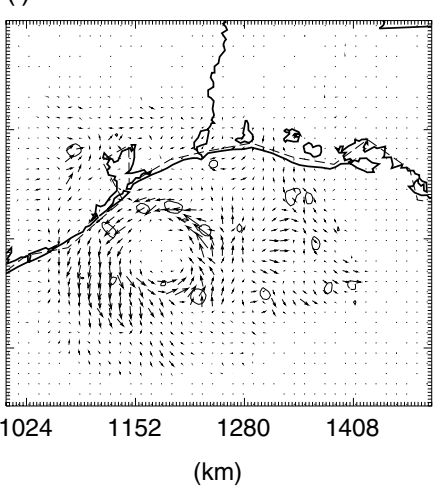

Figure 6. Horizontal wind (vectors) and pressure increments (contours at intervals of $50 \mathrm{~Pa})$ at (a) $-(\mathrm{c})$ the surface, $(\mathrm{d})-(\mathrm{f}) \mathrm{z}=3 \mathrm{~km},(\mathrm{~g})-(\mathrm{i}) \mathrm{z}=6 \mathrm{~km}$ and (j)-(l) $z=9 \mathrm{~km}$, from the first analysis cycle at 0410 UTC 12 August 2008, from ExpVr ((a), (d), (g) and (j)), ExpZ ((b), (e), (h) and (k)), and ExpAll ((c), (f), (i) and (l)). Radars are denoted by the black dots in (a).

cores (Figure 9(e)-(h)). The updraft regions in ExpVr and ExpAll are also deeper and stronger than in ExpZ, with the updrafts centred at roughly 50, 100 and $150 \mathrm{~km}$ from the centre, respectively. In general, the vortex is stronger in ExpZ than in CNTL, while the vortices in ExpVr and ExpAll are the strongest and deepest. Note that part of the predicted vortex is over land and asymmetries develop in the vortex as a result (Figure $8(\mathrm{e})-(\mathrm{h})$ ). The differences among various experiments are probably larger on the east side of the vortex than those between azimuthally averaged fields.
The analyzed and forecast composite reflectivity and horizontal wind vectors at the $3 \mathrm{~km}$ height are presented in Figure 10 for CNTL, ExpVr, ExpZ and ExpAll, together with the observed composite reflectivity (OBS). Stronger and tighter inner core circulations are found in the final analyses of all DA experiments, compared with that in the GFS analysis (Figure 10(a)-(e)). The reflectivity field in ExpVr shows broader and stronger precipitation bands than observations (Figure 10(c)) while the GFS analysis contains no hydrometeor field (Figure 10(b)). ExpAll and ExpZ have 
(a)

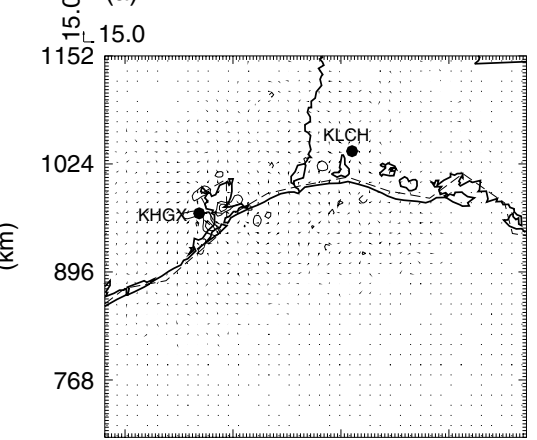

(d)

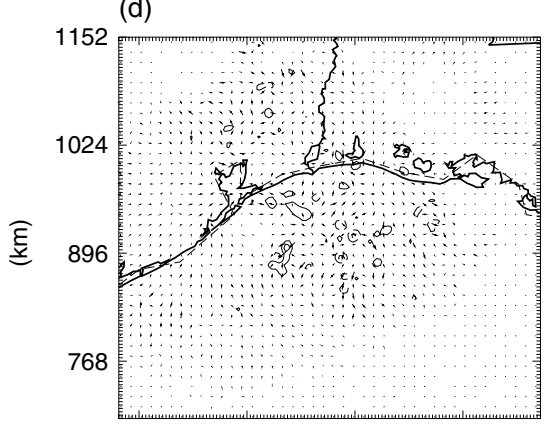

(g)

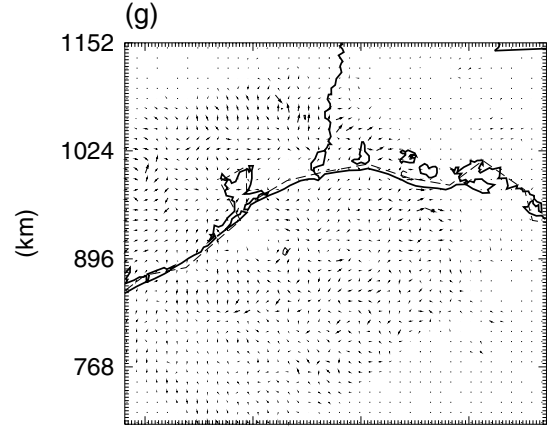

(j)

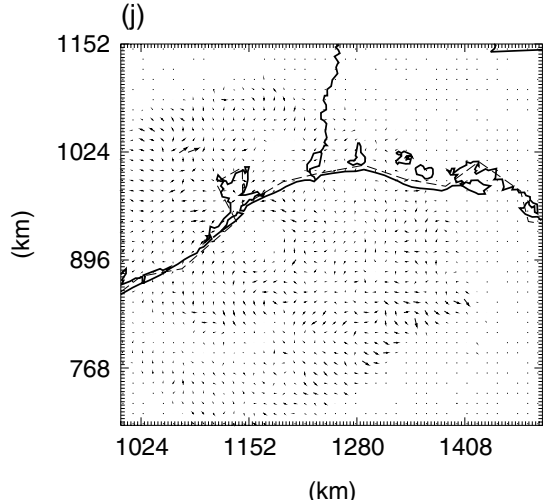

(b)

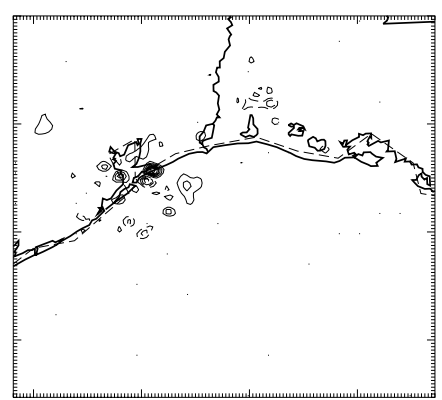

(e)

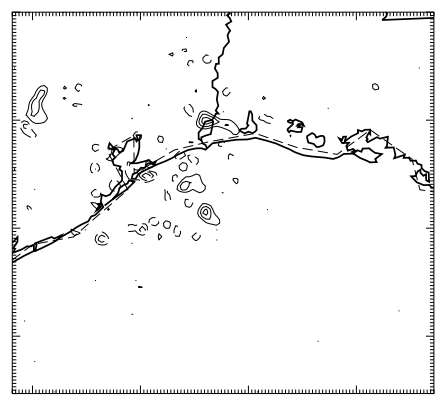

(h)

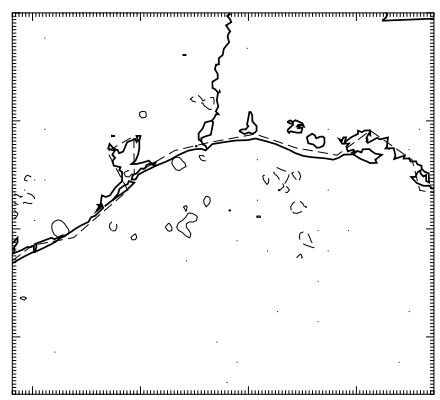

$(\mathrm{k})$

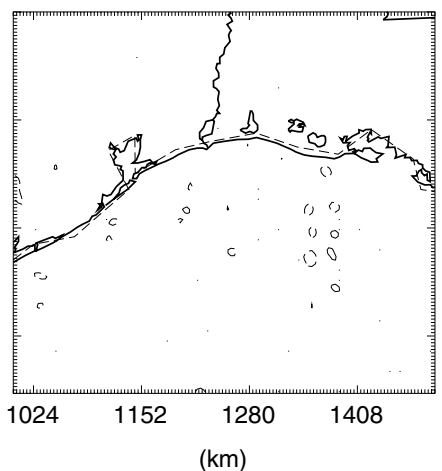

(c)

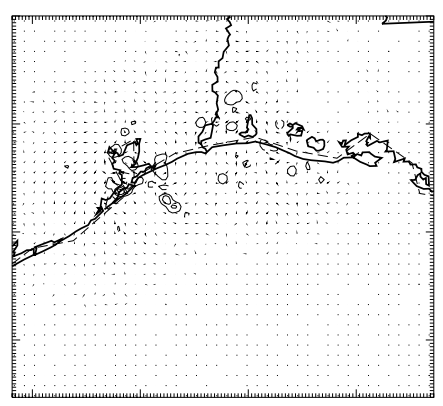

(f)

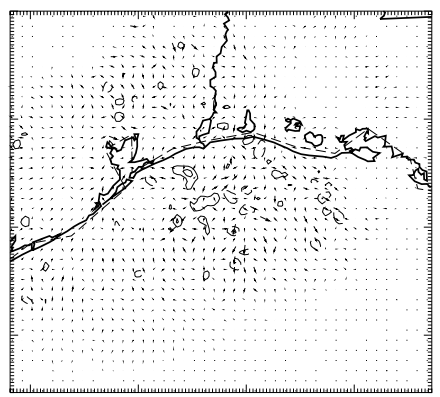

(i)

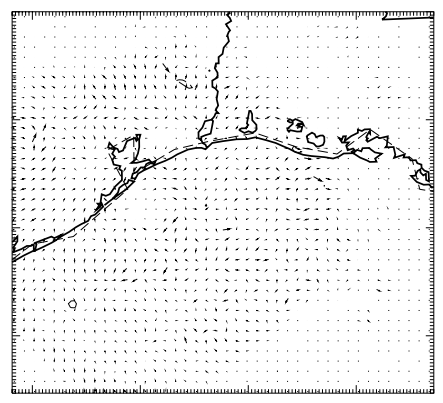

(l)

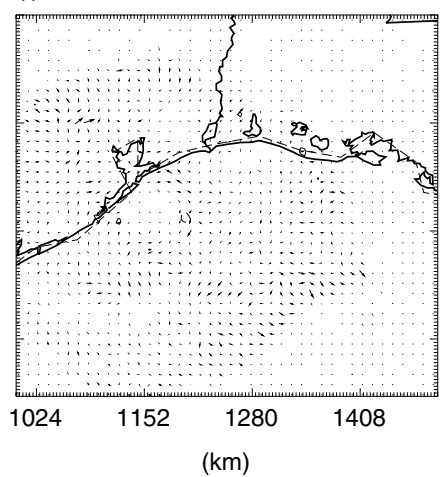

Figure 7. As Figure 6 but for the last analysis cycle at 0600 UTC 13 August 2008.

similar rainband structures and are closer to the observations than ExpVr (Figure 10(d)-(e)). These differences are also reflected in the r.m.s. innovations of $Z$ at this time (Figure 4), where, for both radars, the r.m.s. innovation of $Z$ is about $15 \mathrm{dBZ}$ in ExpVr and only about $5 \mathrm{dBZ}$ in ExpAll and ExpZ. This is not surprising because $Z$ is directly assimilated in ExpAll and ExpZ.

At the $6 \mathrm{~h}$ forecast time, the observed centre of Ike is over land north of Houston. Generally, all experiments with radar DA have a more tightly wrapped rain-band structure that compares better with observations than in
CNTL (Figure 10(f) - (j)). The spiral rain-band northwest of the vortex centre in CNTL is too strong (Figure $10(\mathrm{~g})$ ).

By 1800 UTC or $12 \mathrm{~h}$ into the forecast, Ike has moved further inland and a more asymmetric structure with stronger precipitation in the northwest and southeast quadrants is seen in the observation (Figure 10(k)). A more elongated precipitation pattern is exhibited by CNTL with a clear-air hole at the vortex centre that is not observed (Figure 10(1)). With radar data, the precipitation patterns are closer to the observations, with the vortex centre filled with weak precipitation (Figure $10(\mathrm{~m})-(\mathrm{o}))$. Among the radar DA experiments, ExpZ has a precipitation pattern that 


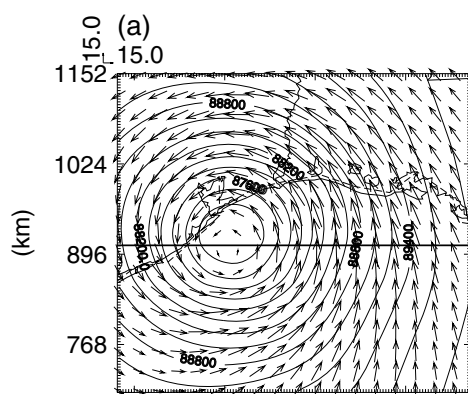

(b)

(c)

(d)
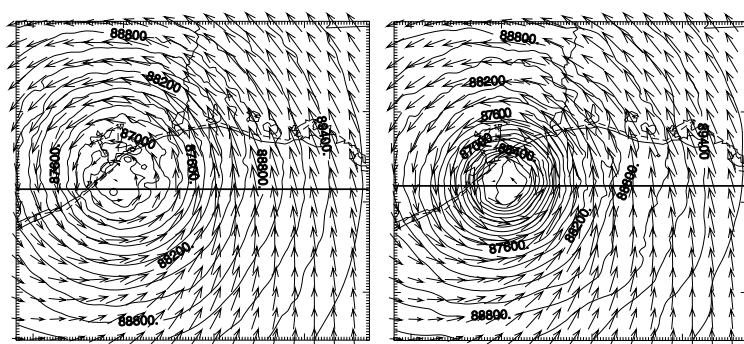

(g)

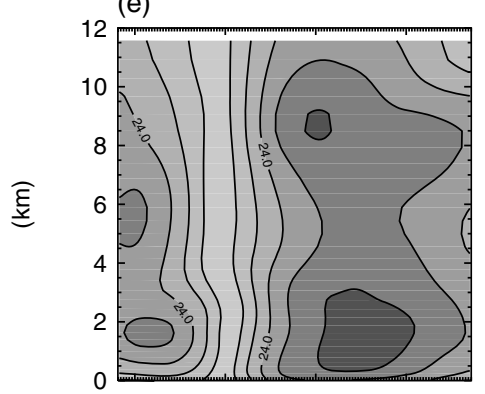

(f)
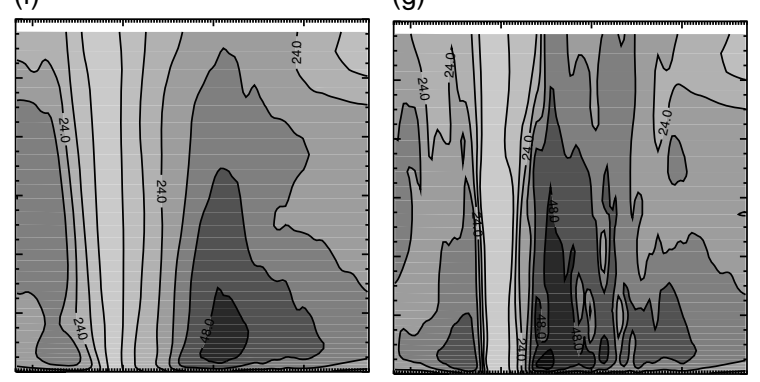

(k)

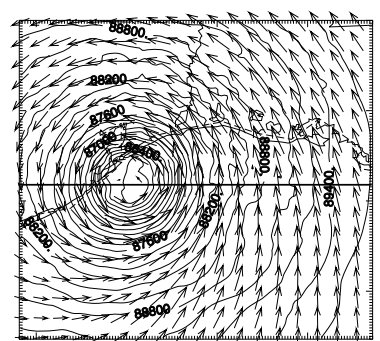

(h)

(j)
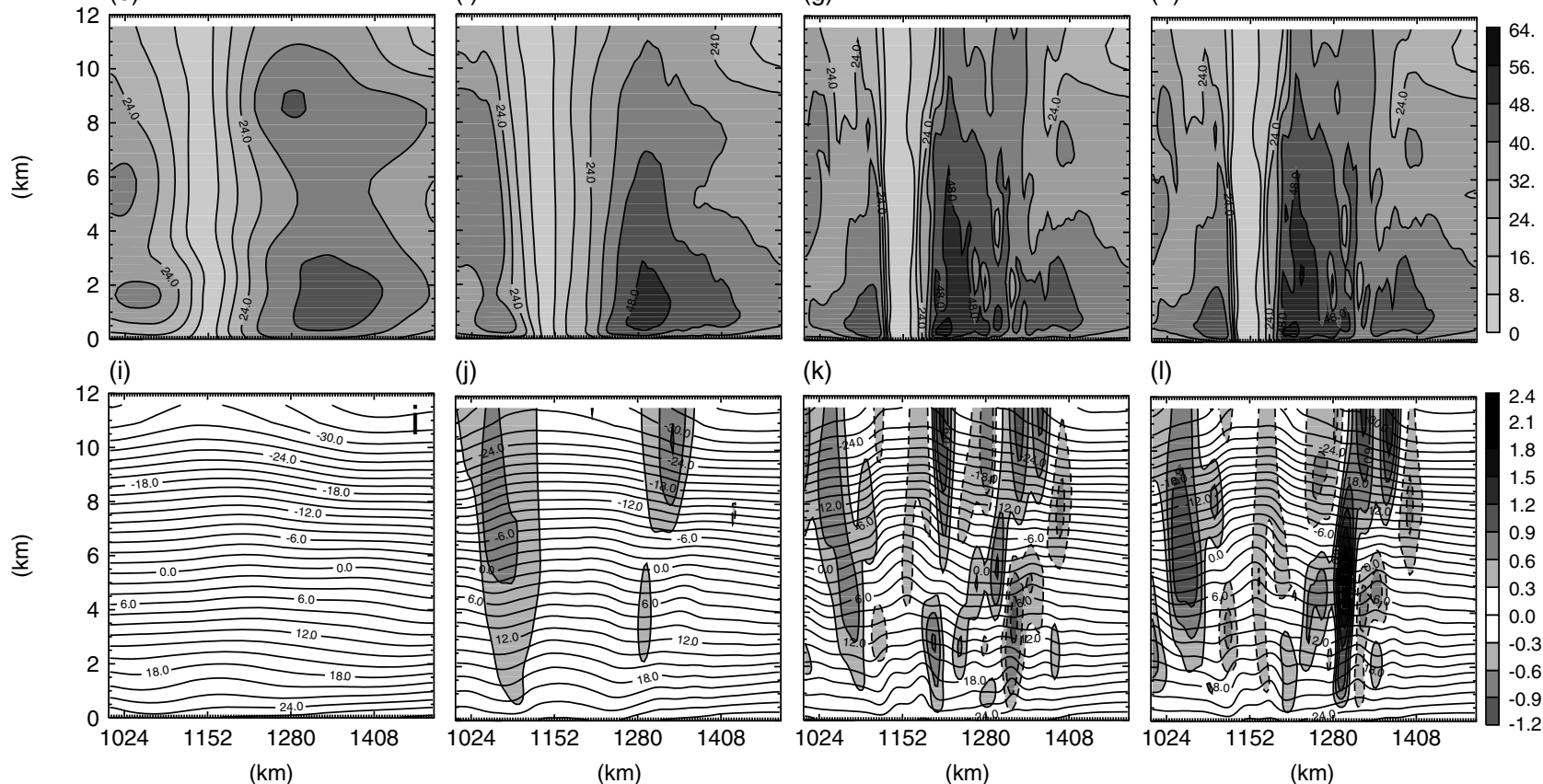

(I)

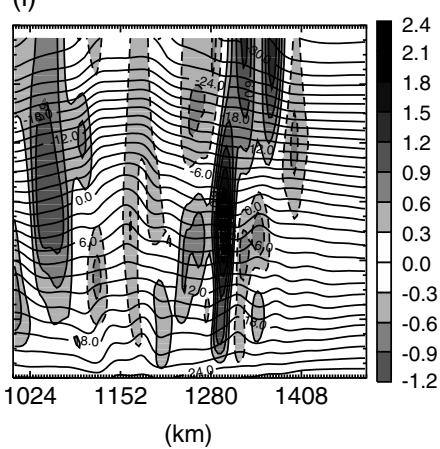

Figure 8. (Top panels) The horizontal wind (vectors) and pressure (contours at intervals of $20 \mathrm{~Pa}$ ) at $1 \mathrm{~km}$ height and 0600 UTC from (a) the GFS analysis, and analyses of (b) ExpZ, (c) ExpVr and (d) ExpAll. (Middle panels) The east-west cross-section of horizontal wind speed through the vortex centre of each experiment (indicated by the black lines in (a)-(d)) for the analyses of GFS, ExpZ, ExpVr and ExpAll, respectively. (Lower panel) The same east-west cross-section for temperature (contours) and vertical velocity (shaded contours) for the four corresponding cases.

(a)

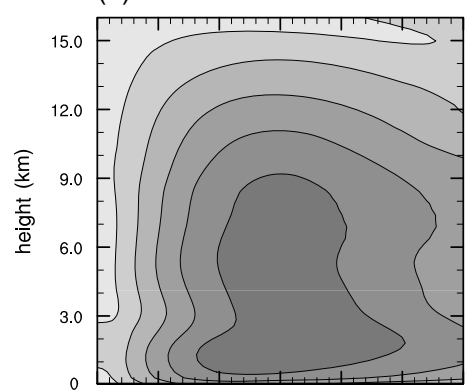

(e)

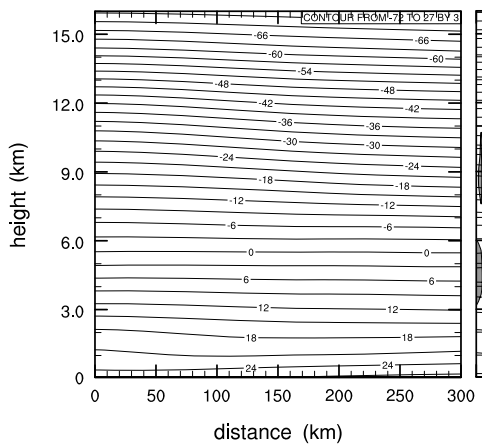

(b)

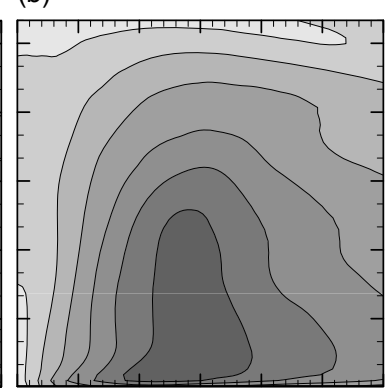

(f)

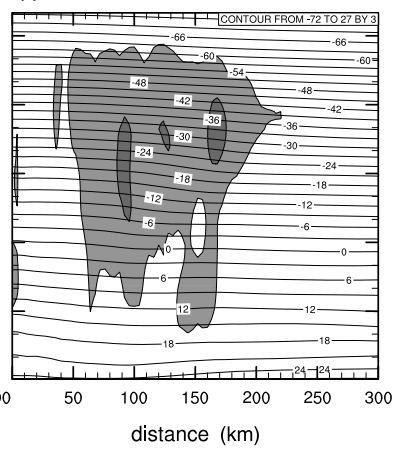

(c)

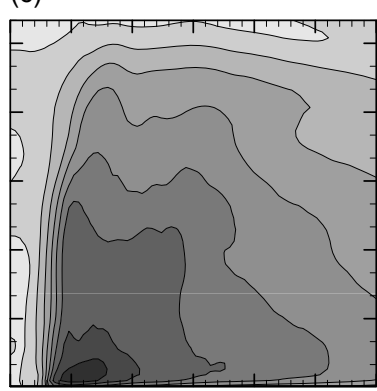

(g)

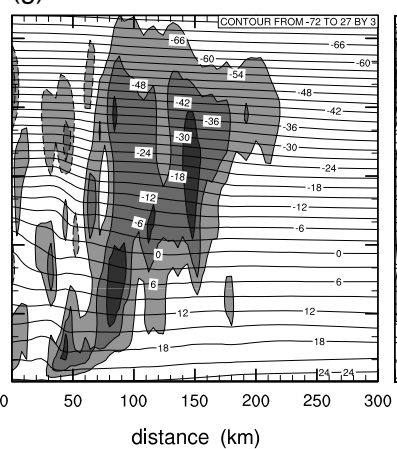

(d)

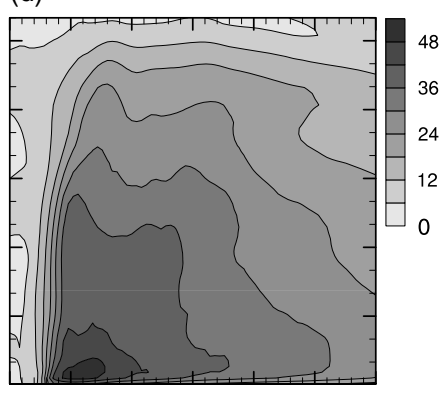

(h)

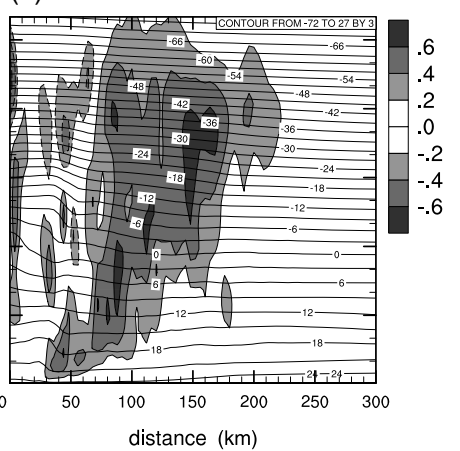

Figure 9. Radius-height plots of azimuthal mean horizontal velocity fields (a)-(d), vertical velocity (shaded contours in (e)-( h)) and temperature (contours in (e)-(h)) at 0600 UTC for (a, e) CNTL, (b, f) ExpZ, (c, g) ExpVr and (d, h) ExpAll. 
(a)

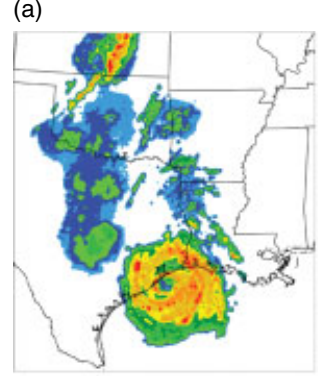

(b)

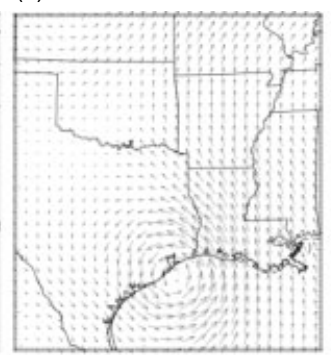

(f)

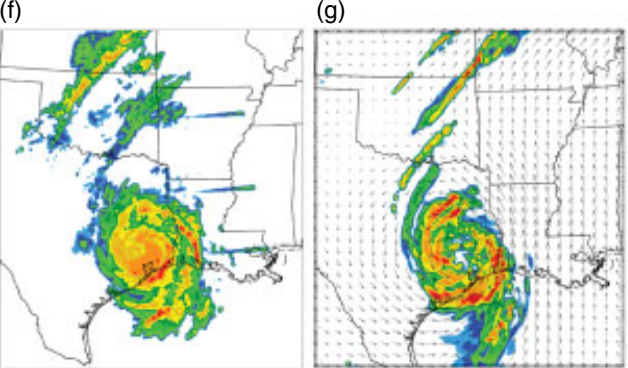

(k)
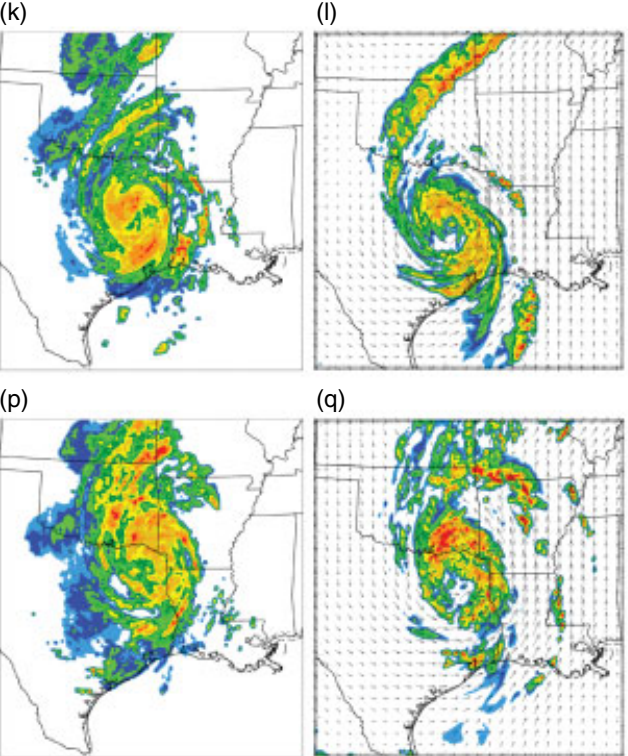

(c)

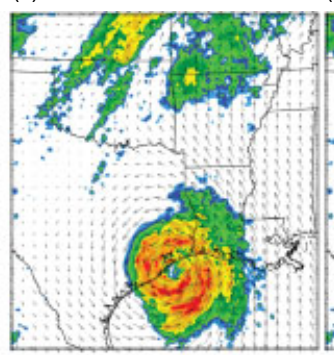

(d)

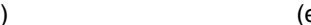

(e)

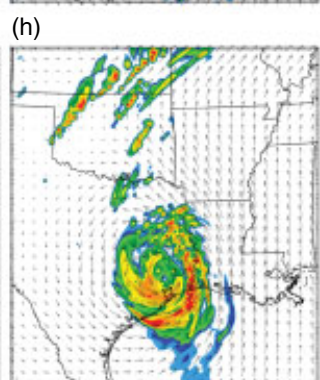

(m)

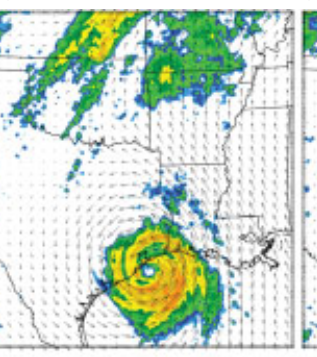

(

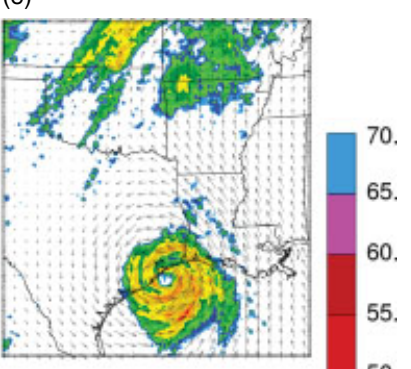

(j)

50.

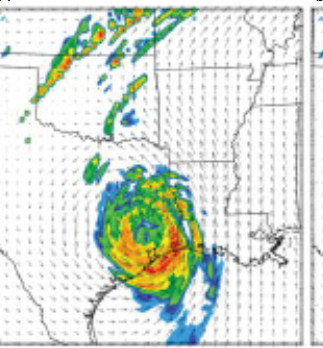

(n)

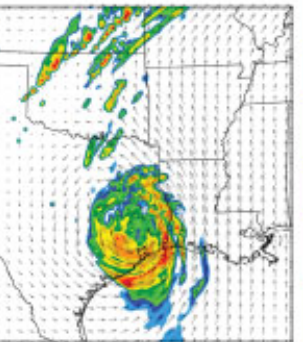

45.

35.

30.

25.

20.

(o)

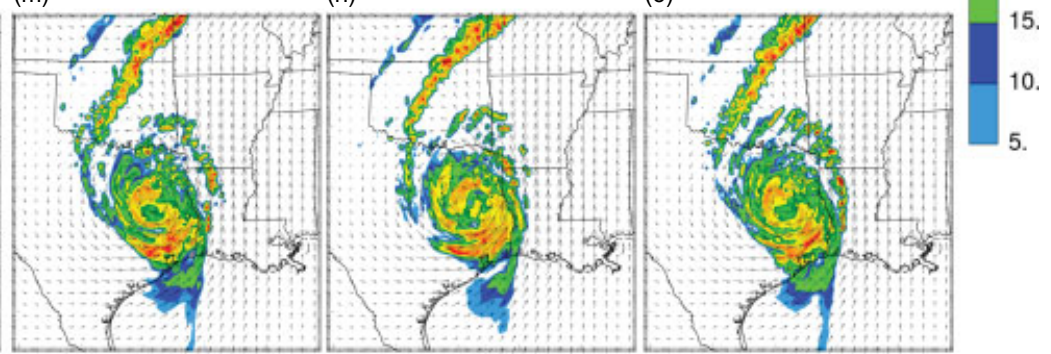

( $r)$

(s)

(t)

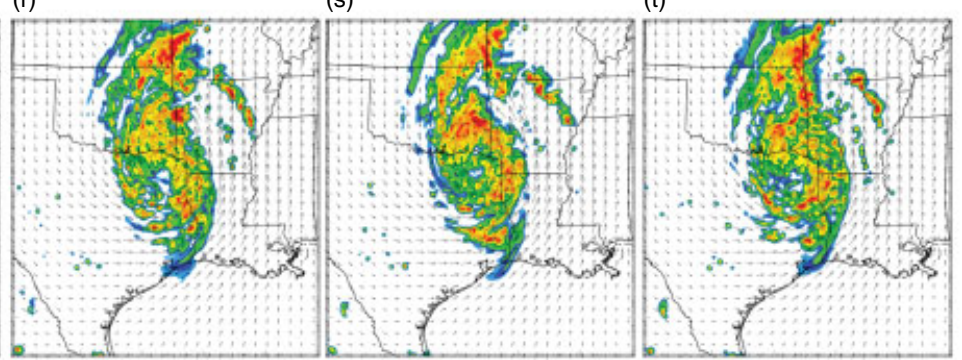

Figure 10. Composite reflectivity (colour shaded) and wind vectors at $3 \mathrm{~km}$ height analyzed and predicted by experiments ((b), (g), (l) and (q)) CNTL, $((\mathrm{c}),(\mathrm{h}),(\mathrm{m})$ and $(\mathrm{r})) \operatorname{ExpVr},((\mathrm{d}),(\mathrm{i}),(\mathrm{n})$ and $(\mathrm{s})) \operatorname{ExpZ}$, and ((e), (j), (o) and (t)) ExpAll, as compared with ((a), (f), (k) and (p)) corresponding observations. The times shown are 0600, 1200, 1800 UTC 13 September and 0000 UTC 14 September 2008, for the four rows from top to bottom.

appears closest to the observations (Figure 10(n)). At this time, all forecasts show a rain band oriented southwest to northeast through Oklahoma that is apparently the result of interaction between the hurricane circulation and a midlatitude cold front. This rain band, however, is overpredicted in the model compared with the observations.

At the final forecast time of 0000 UTC 14 September, the strongest precipitation is now mostly north of Texas. The interaction with the cold front to the north and the moisture transport from the Gulf of Mexico leads to rainfall in Oklahoma and Arkansas and a more asymmetric precipitation pattern around the vortex centre overall (Figure 10(p)). CNTL still exhibits a precipitation-free hole in the vortex centre (Figure 10(q)) while the vortex centres in ExpZ and ExpAll are filled with precipitation (Figure 10(s) and Figure 10(t)). The overall precipitation patterns in the radar-assimilating experiments are arguably closer to the radar observations at this time, especially in the outer precipitation region in northeast Oklahoma and southwest
Kansas. Quantitative verifications of precipitation will be given later.

\subsection{Intensity and track forecasting}

The MSLPs every $3 \mathrm{~h}$ during the $18 \mathrm{~h}$ long forecasts from all experiments are plotted in Figure 11(a), along with the best-track MSLP from the National Hurricane Center. All radar DA experiments show clear improvement compared with CNTL during the first $12 \mathrm{~h}$ of forecast for intensity based on MSLP. The analyzed MSLPs of about $955 \mathrm{hPa}$ in ExpVr and ExpAll (with two curves almost overlapping) at 0600 UTC are much lower than the $975 \mathrm{hPa}$ of CNTL, although still somewhat higher than the best-track value of $951 \mathrm{hPa}$. Assimilation of $Z$ alone leads to a much smaller improvement of $8 \mathrm{hPa}$ over CNTL, yielding a weaker vortex than when assimilating $V_{\mathrm{r}}$ or both $V_{\mathrm{r}}$ and $Z$. The analysis intensity improvement over CNTL is about $83 \%$ for ExpVr and ExpAll and about 33\% for ExpZ in terms of MSLP error reduction, defined as $\left(\right.$ Error $_{\mathrm{CNTL}}-$ Error $\left._{\mathrm{DA}}\right) /$ Error $_{\mathrm{CNTL}}$. It is 

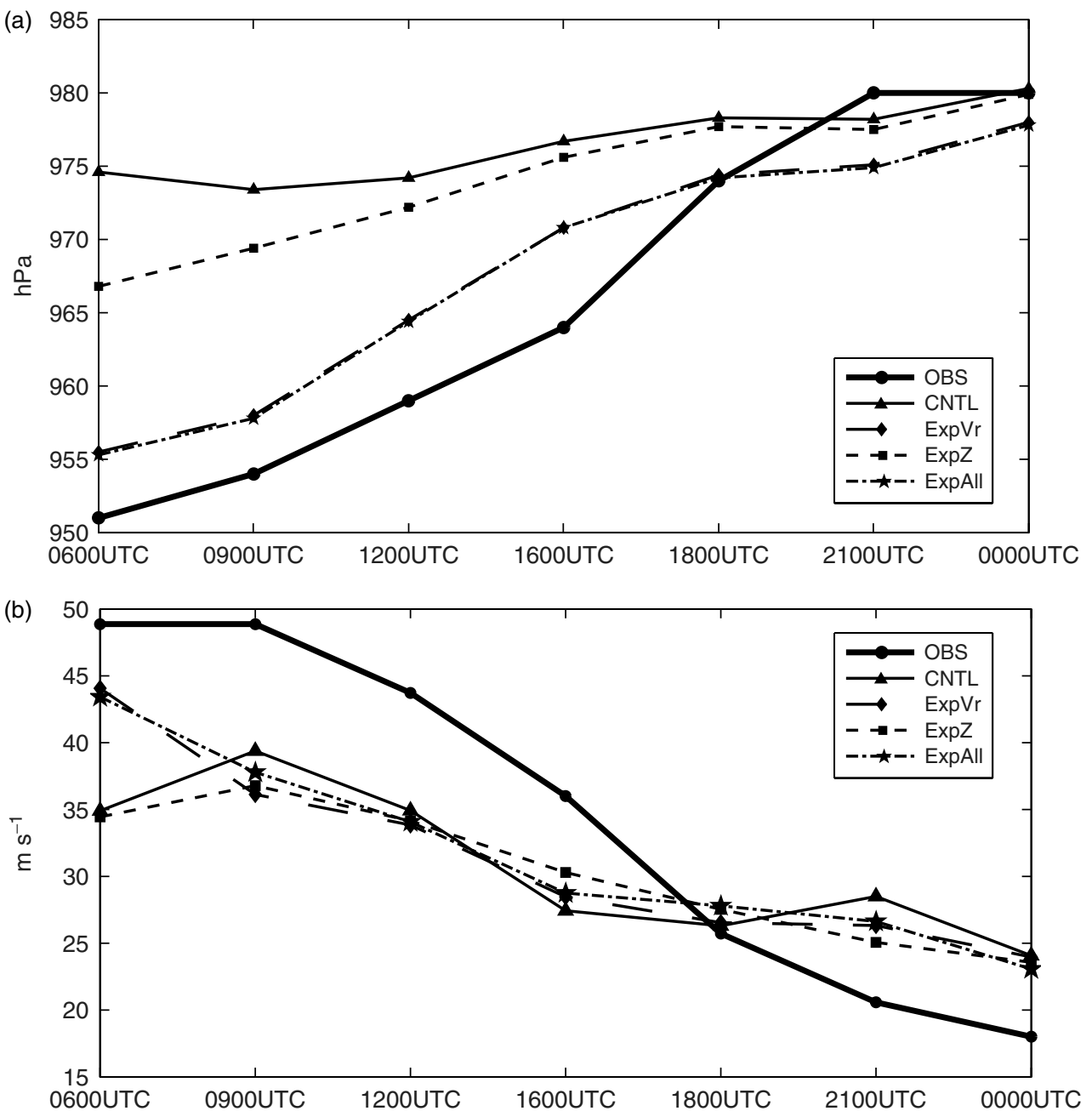

Figure 11. (a) The predicted minimum sea-level pressures and (b) maximum surface wind speed for Hurricane Ike, plotted every 3 h from 0600 UTC 13 September to 0000 UTC 14 September, compared with the best-track values ( OBS).

noted that for convenience the term 'error' is used here to describe the difference between the model results and the best-track data, even though the latter typically also contain error.

The MSLP in CNTL of about $975 \mathrm{hPa}$ is too high and does not change much during the first $12 \mathrm{~h}$ of the forecast, while the observed hurricane starts at about $951 \mathrm{hPa}$ MSLP and continues to weaken until 2100 UTC, due to landfall shortly after 0600 UTC. ExpVr and ExpAll capture the pressure rise at rates similar to the best-track data before 1500 UTC, but their rate of rise is slower than observed between 1500 and 2100 UTC. This discrepancy might have been caused by prediction model error, such as in the treatment of surface processes responsible for the vortex spin down. The slower spin down resulted in a lower MSLP after 1800 UTC than observed. A similar behaviour was also observed in the study of Zhao and Xue (2009), which used the ARPS $3 \mathrm{D}-$ Var and cloud analysis system to assimilate radar data for the same case. Figure 11 also shows that the assimilation of reflectivity data in addition to radial velocity in ExpAll did little to MSLP, with differences between the analysis and forecast MSLPs of ExpAll and ExpVr being generally less than $0.5 \mathrm{hPa}$. Comparing further with the results of ExpZ shows clearly that the assimilation of $V_{\mathrm{r}}$ data provides most of the impact on MSLP analysis and forecasting. This result is different from that of Zhao and Xue (2009) where adjustments to temperature and moisture fields through a complex cloud analysis package can greatly affect MSLP.

The predicted maximum surface wind speeds are also plotted in Figure 11(b), along with the best-track data for comparison. The maximum surface winds of ExpVr and ExpAll at 0600 UTC are clearly higher than that from GFS analysis (CNTL). Even though the vortex in ExpZ is clearly stronger than that in CNTL, as indicated by the azimuthally averaged wind fields (Figure 9(a) and (b)), the maximum surface wind in ExpZ is slightly weaker than in CNTL. During the forecast, the maximum surface winds of all simulations are closer to each other and weaker than the best track.

The predicted tracks and track errors are plotted in Figure 12, along with the best track (OBS). Starting from a quite small initial track error of $7 \mathrm{~km}$, CNTL takes the westernmost path during the $18 \mathrm{~h}$ long forecast, which is the furthest from the observed track among all experiments. Its error increases with forecast time and reaches $80 \mathrm{~km}$ at 0000 UTC 14 September (Figure 12(b)). With radar DA, the track errors in the final analyses at 0600 UTC are all larger than in CNTL. These larger errors are partly related to the way the centre location is determined. The initial centre location is determined from the mean pressure of the 32-member ensemble. After the averaging, the mean pressure field shows a somewhat elongated vortex, creating 

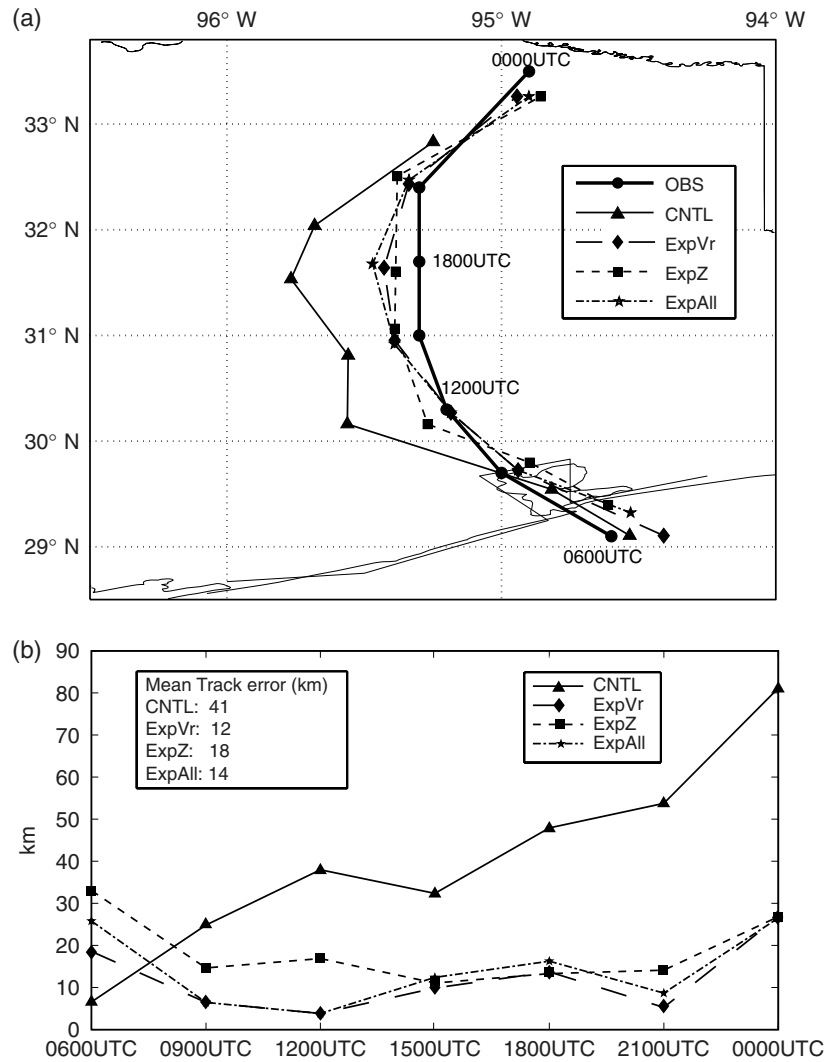

Figure 12. (a) The predicted track for Hurricane Ike, plotted every $3 \mathrm{~h}$ from 0600 UTC 13 September to 0000 UTC 14 September, compared with the best track (OBS). (b) The predicted track error for Hurricane Ike, plotted every $3 \mathrm{~h}$ from 0600 UTC 13 September to 0000 UTC 14 September. To highlight the difference between the tracks, the map in (a) is stretched along the east-west direction (latitude).

some difficulty in determining the exact location of the vortex centre. For ExpAll, averaging the centre locations of individual members results in a mean centre location that is $10 \mathrm{~km}$ closer (see Figure 16(a)).

In the first $3 \mathrm{~h}$ of forecast, the hurricane in CNTL moves slower than in all DA experiments. At 0900 UTC after the landfall, the predicted tracks in all DA experiments are closer to the best track than in CNTL, with the track errors all being less than $20 \mathrm{~km}$ (Figure 12(b)) while those of ExpVr and ExpAll are less than $10 \mathrm{~km}$. From 1200 to 2100 UTC, the observed hurricane took a mostly northward path toward Tyler, Texas $\left(32.3^{\circ} \mathrm{N}, 95.3^{\circ} \mathrm{W}\right)$. The ExpZ experiment captures this northward direction very well during these $9 \mathrm{~h}$ but with a westward displacement of 15 to $18 \mathrm{~km}$. Both ExpVr and ExpAll have very small track errors of less than $5 \mathrm{~km}$ at 1200 UTC but take a more westward curved path than ExpZ between 1200 and 2100 UTC. All three DA experiments have southward displacement error of about $27 \mathrm{~km}$ at 0000 UTC September 14, the end of the $18 \mathrm{~h}$ forecast. Although ExpZ has a larger mean track error of $18 \mathrm{~km}$ compared with ExpVr and ExpAll (Figure 12(b)), it is encouraging to see that assimilating $Z$ alone still results in $56 \%$ improvement on average compared with CNTL in track forecasting.

$\mathrm{Pu}$ et al. (2009) observed a marginal improvement of the Hurricane Dennis (2005) intensity forecast when assimilating reflectivity data alone using the WRF 3D-Var (Xiao et al., 2007). They also found that the impact from reflectivity data alone on intensity forecast is smaller than assimilating radial velocity data alone or assimilating both radial velocity and reflectivity data. They attributed part of this to the small impact of reflectivity data on the track forecast. In our case, the hurricane centre in the analysis of ExpZ at 0600 UTC is too far north near the coast, and the larger position error than in ExpVr and ExpAll continued until 1500 UTC (Figure 12(a) and (b)), but the error is still much smaller than in CNTL.

In our case, the assimilation of radial velocity clearly has a larger impact on intensity than assimilating $Z$ alone. This may not be surprising because hurricanes are winddominated systems; radial velocity data from two Doppler radars can measure the wind structures within the hurricanes quite well, while EnKF updates pressure and thermodynamic fields through cross covariances and through dynamic mass-wind adjustment during the assimilation cycles. The reflectivity data are directly linked to the microphysical fields, while the correlations between reflectivity and wind fields, as estimated from the ensemble, may be less certain. In fact, updating the latter variables in the EnKF degrades the analysis, as pointed out in section 2.4, and is therefore not performed in the experiments presented here. More effective assimilation of reflectivity data using EnKF, especially for tropical storms, will require more research.

\subsection{Precipitation forecasting}

Flooding from local precipitation and the storm surge caused by Ike was one of the major causes of death and property loss, highlighting the importance of QPF for landfalling hurricanes (Rogers et al., 2009). Figure 13 shows the $18 \mathrm{~h}$ accumulated precipitation for all experiments along with the stage IV precipitation data (Lin and Mitchell, 2005). The observed rainfall maximum is positioned around Huntsville $\left(30.7^{\circ} \mathrm{N}, 95.55^{\circ} \mathrm{W}\right)$ and Conroe $\left(30.3^{\circ} \mathrm{N}\right.$, $95.45^{\circ} \mathrm{W}$ ), Texas, north of Houston (Figure 13(a)). The CNTL experiment fails to predict this maximum completely (Figure 13(b)). Assimilation of radar data in ExpVr, ExpZ and ExpAll helps to capture this intense precipitation area, although the strength and coverage are underpredicted (Figure 13(c)-(e)). For lighter or stratiform precipitation it is not easy to tell which experiment has a better prediction.

To quantify the precipitation forecast skills, equitable threat scores (ETS hereafter; Schaefer, 1990) against the stage IV data for $3 \mathrm{~h}$ accumulated precipitation are calculated and plotted for all experiments in Figure 14. A threshold of $30 \mathrm{~mm}$ is chosen to represent convective rainfall. In the first $6 \mathrm{~h}$ of forecast, all experiments with radar data have higher ETS scores than CNTL. From 1200 UTC to 1800 UTC, the score is still higher in ExpZ while those of ExpAll and ExpVr become close to that of CNTL. There are ETS increases for ExpAll and ExpVr from 1800 to 2100 UTC. The track error reductions in ExpVr and ExpAll during this period may have contributed to the ETS increase. At the end of forecasts, ETSs of all the experiments are below 0.1, partly due to the shrinking area of convective precipitation at this time; in such a case, small position errors with convective cores can lead to very low scores. The ETS is also generally lower for smaller fractional coverage.

The ETS of $18 \mathrm{~h}$ accumulated precipitation is also calculated (Figure 15) for four thresholds ranging from $30 \mathrm{~mm}$ to $120 \mathrm{~mm}$. Lower (higher) thresholds represent precipitation of more stratiform (convective) nature. For all the thresholds, radar DA clearly helps improve QPF. The relative improvement is larger for larger thresholds, implying 
(a)

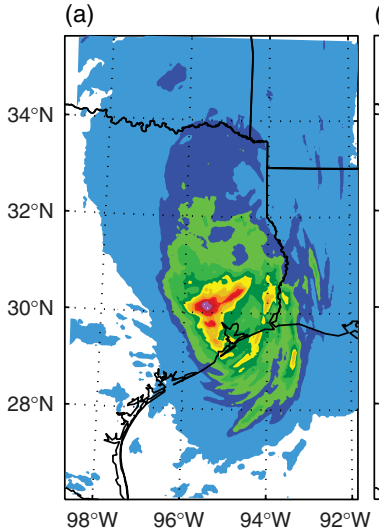

(b)

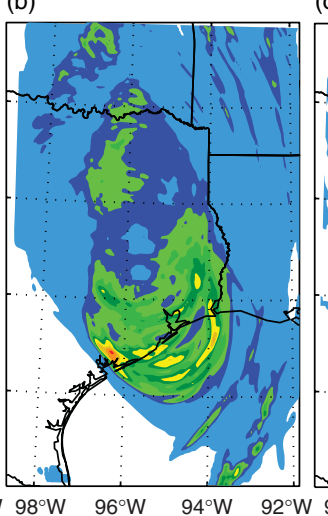

(c)

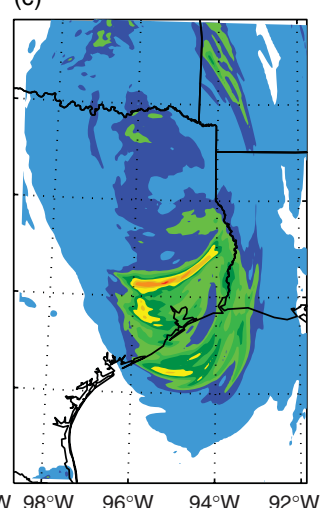

(d)

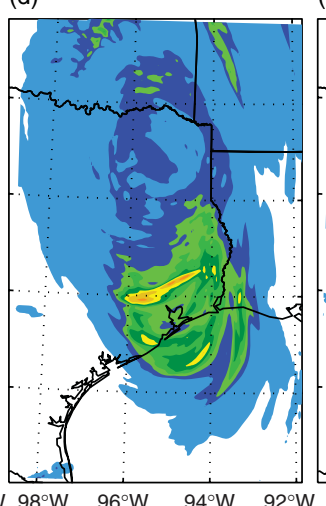

(e)

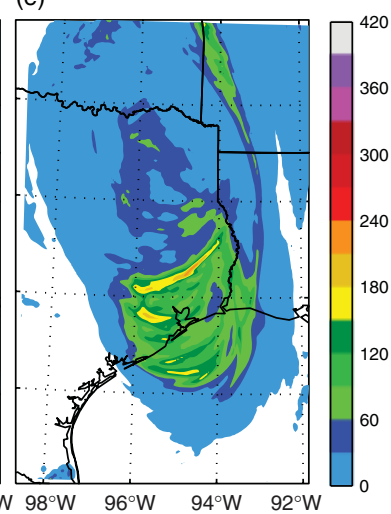

Figure 13. Eighteen-hour accumulated precipitation forecast from 0600 UTC 13 September to 0000 UTC 14 September for (a) observations, (b) CNTL, (c) ExpVr, (d) ExpZ and (e) ExpAll.

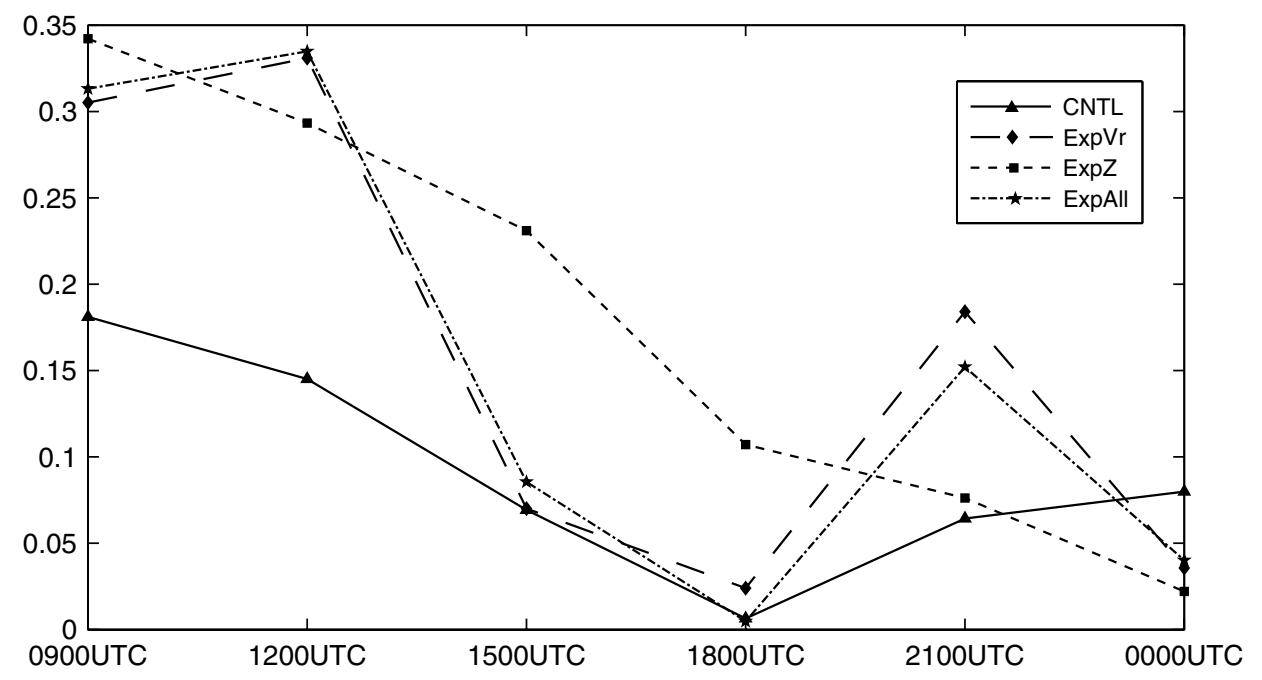

Figure 14. Equitable threat scores (ETS) of $3 \mathrm{~h}$ accumulated precipitation at the $30 \mathrm{~mm}$ threshold for CNTL, ExpVr, ExpZ and ExpAll.

more impact of the radar data on convective precipitation. For the $120 \mathrm{~mm}$ threshold, the improvements of the three radar DA experiments are all about 300\% relative to CNTL, or the ETSs are about four times that of CNTL. It should be noted that storm track plays an important role in the precipitation forecast. In our study, the effect of track error is not separated from the precipitation pattern error. Future work can look into this important aspect (Marchok et al., 2007).

\section{Ensemble forecasts}

\subsection{Ensemble forecasts of intensity and track}

The EnKF DA provides an ensemble of analyses that can be used to initialize an ensemble of forecasts. Ensemble forecasts of 32 members are carried out from the final analyses of ExpAll at 0600 UTC. At this time, the ensemble spread of MSLP is $0.7 \mathrm{hPa}$, rather small compared with the intensity error of $4 \mathrm{hPa}$ (not shown). This may be because of the large number of radar observations constraining the vortex intensity rather strongly, and/or because of the relatively small forecast spread growth during the assimilation period due to the lack of synoptic to largescale uncertainties in the hurricane environment in our experiments and the relatively short forecast lengths during the analysis cycles.

During the later forecast, the intensity spread does not increase much with time; this is at least partly because Ike is in its weakening stage. Other studies have also found that the intensity error growth of a decaying hurricane is not as large as intensifying hurricanes and the ensemble spread tends to decrease with time (see, e.g. figure 12 of Zhang et al., 2009). The ensemble mean forecast MSLP is similar to that of the corresponding deterministic forecast.

The ensemble forecast tracks and ensemble track spreads are plotted in Figure 16. The deterministic forecast track starting from the ensemble mean analysis and the mean track of ensemble forecasts are also shown for comparison. Uncertainty growth is observed in the ensemble track forecasts (Figure 16(a)). The spread of the ensemble track is defined as

$$
\sqrt{\frac{1}{N-1} \sum_{i=1}^{N} d_{i}^{2}},
$$

where $N$ is the ensemble size and $d_{\mathrm{i}}$ is the distance between the vortex centres of the $i$ th member and the mean. The track spread increases during most of the forecast time except for a temporary reduction in the first $3 \mathrm{~h}$ (Figure 16(b)). The track spread at the end of $18 \mathrm{~h}$ forecast is increased by 


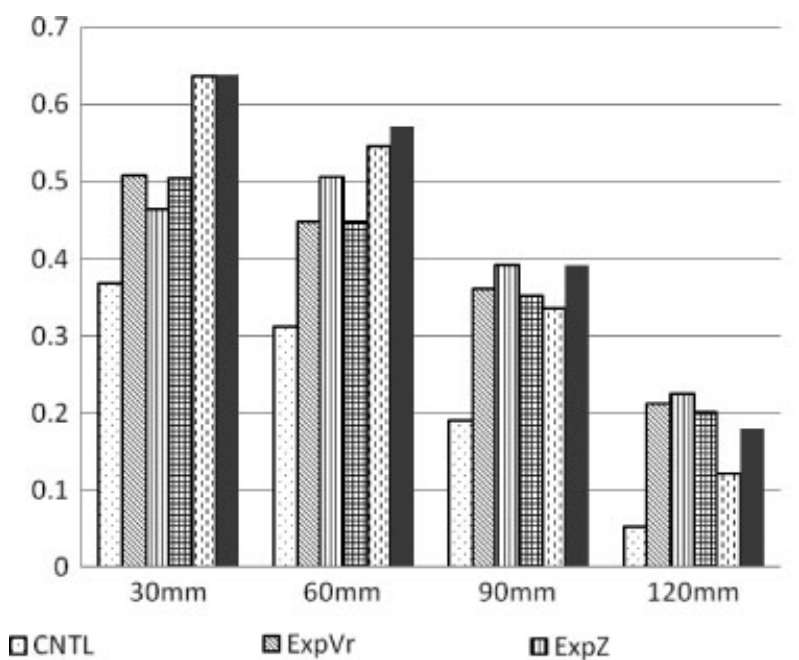

田ExpAll (Det. Fcst) QExpAll (Ens. Mean) ExpAll(Prob. Matching)

Figure 15. Equitable threat scores (ETSs) of $18 \mathrm{~h}$ accumulated precipitation from 0600 UTC 13 September to 0000 UTC 14 September, at the threshold of $30 \mathrm{~mm}, 60 \mathrm{~mm}, 90 \mathrm{~mm}$ and $120 \mathrm{~mm}$ for deterministic forecasts of CNTL, ExpVr, ExpZ and ExpAll, and the ensemble mean forecast and the probability matched ensemble mean forecast of ExpAll.

(a)
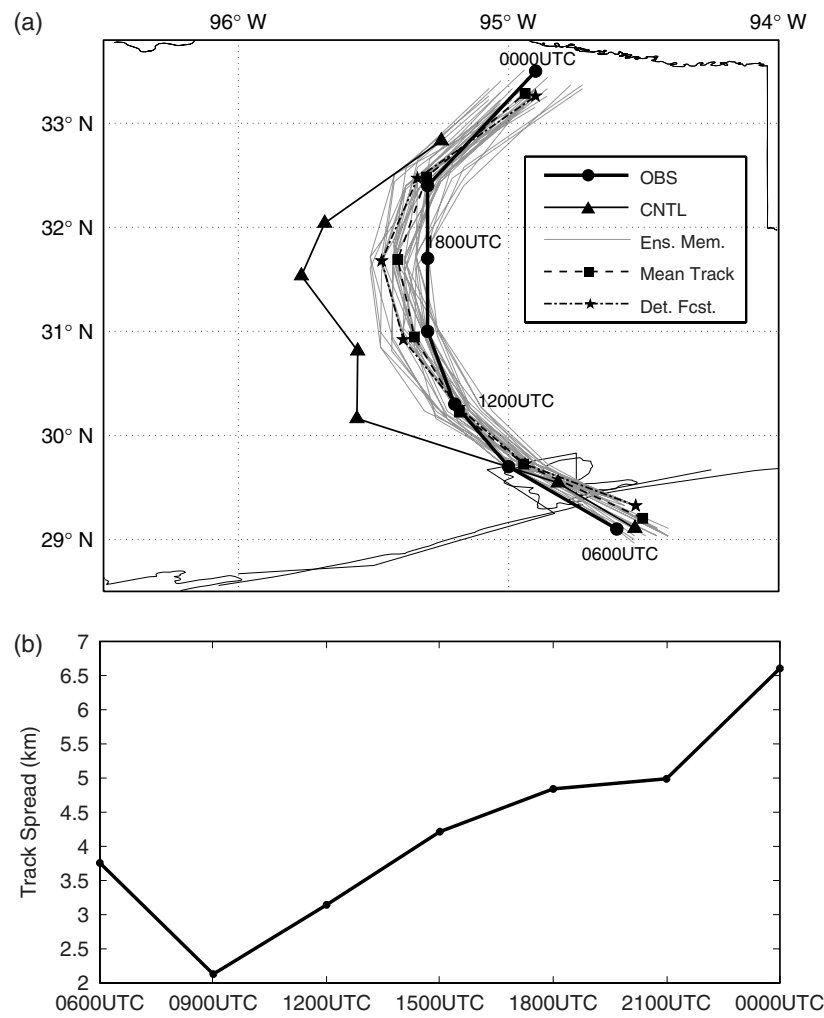

Figure 16. (a) The ensemble forecast tracks for ExpAll (grey), compared with the best track, CNTL, mean track of ensemble and deterministic track forecasts. (b) The spread of ensemble tracks for ExpAll.

$75 \%$ compared with the beginning. As mentioned in section 4.2 , the average vortex position of ensemble members is closer to the best track than that of the ensemble mean analysis at 0600 UTC. The mean predicted track of the ensemble members also has certain improvement over the deterministic forecast at 1500 and 1800 UTC (Figure 16(a)), suggesting the potential benefit of the mean ensemble forecast track over the deterministic track forecast.

\subsection{Ensemble forecasts of precipitation}

Predicted ETSs are also calculated for each ensemble member of ExpAll (Figure 17). At most forecast times, most of the ensemble members have higher scores than CNTL. The ETSs of the deterministic forecast always fall within the range of ensemble ETSs during the $18 \mathrm{~h}$ of forecast. The ETS of the ensemble mean is higher than the deterministic forecast at 2100 UTC, and comparable or lower at other forecast hours.

In high-resolution predictions, the precipitation field tends to have high spatial and temporal variations across the ensemble members. One problem with the simple ensemble mean of precipitation is the excessively broad rainfall areas and rainfall maxima underestimation owing to the smoothing effect of averaging (Kong et al., 2009). The simulated radar reflectivity of the ensemble mean is plotted in Figure 18, compared with the observations and deterministic forecast. The averaging over ensemble members leads to a wider region of weak rainfall surrounding the vortex centre than the deterministic forecast at the displayed times, while the magnitude of strong reflectivity is generally too low. In our study, the ETS of $18 \mathrm{~h}$ accumulated rainfall for the ensemble mean is larger than the corresponding deterministic forecast at the $30 \mathrm{~mm}$ and $60 \mathrm{~mm}$ thresholds (Figure 15), benefiting from the higher spatial coverage. At the $90 \mathrm{~mm}$ and $120 \mathrm{~mm}$ thresholds, however, the ETSs of the simple ensemble mean are lower than those of the deterministic forecast (Figure 15); much of this is due to the excessive smoothing associated with the ensemble averaging, thereby reducing the area coverage of heavy precipitation.

Probability matching (PM, hereafter) provides a useful way to reorganize the precipitation fields among ensemble members and improve the QPF. Probability matching sets the probability distribution function (PDF) of the less accurate dataset to be the same as that of the more accurate dataset (Ebert, 2001). The PM method in this study follows Ebert (2001) by assuming that the best spatial distribution of the rainfall field is represented by the ensemble mean, while the best frequency distribution is given by the ensemble QPFs. The PM algorithm first combines the accumulated precipitation from all the ensemble members on the selected domain and ranks the precipitation from the greatest to the smallest to obtain the PDF of the accumulated precipitations. This ensemble of precipitation is saved as array 1. Similarly, the precipitation of the ensemble mean over the same domain is also ranked in highest to the lowest order, saved as array 2. Then the grid point with the highest value in array 2 is reassigned to the highest precipitation in array 1 , and so on. The grid point with the $m$ th value in array 2 is reassigned to the $(N \times m)$ th value in array 1 , where $N$ is the ensemble size. The PM method has been applied successfully in Clark et al. (2009) and Kong et al. (2009) for post-processing convective-scale ensemble precipitation forecasts.

With PM, the $3 \mathrm{~h}$ accumulated rainfall ETSs at the $30 \mathrm{~mm}$ threshold have a slight but clear improvement over the regular ensemble mean for all forecast times (Figure 17). For the $18 \mathrm{~h}$ accumulated precipitation, PM has higher ETS scores at the $60 \mathrm{~mm}, 90 \mathrm{~mm}$ and $120 \mathrm{~mm}$ thresholds than the regular mean (Figure 15). At the $120 \mathrm{~mm}$ threshold, representing strong rainfalls, the PM processing increases the simple mean score by about 50\%. Among the three sets of scores for experiment ExpAll, the PM-processed 


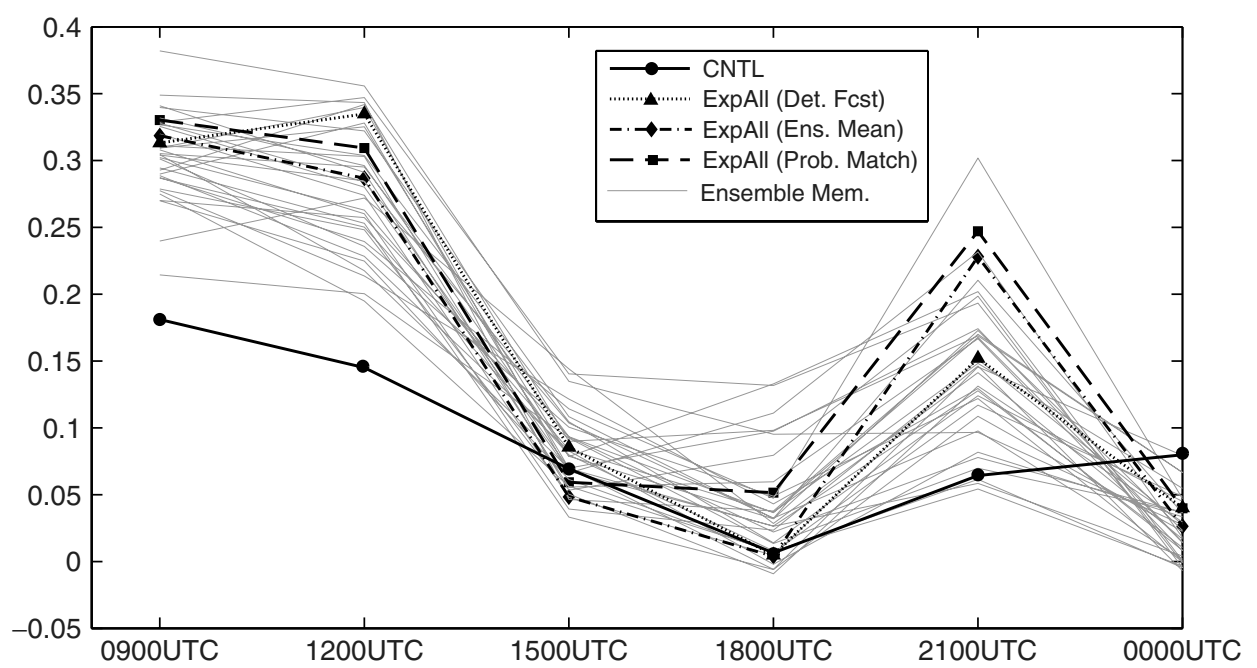

Figure 17. Equitable threat scores of $3 \mathrm{~h}$ accumulated precipitation at the $30 \mathrm{~mm}$ threshold for the ensemble forecasts (grey), the deterministic forecast, the ensemble mean forecast and the probability matched ensemble mean forecast of ExpAll.

(a)

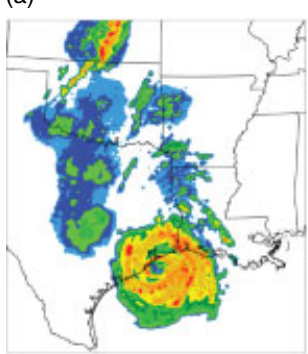

(e)

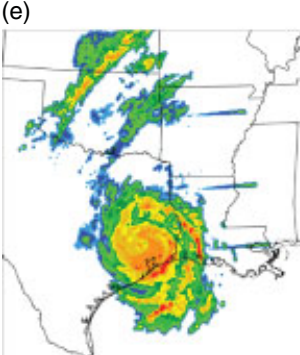

(i)

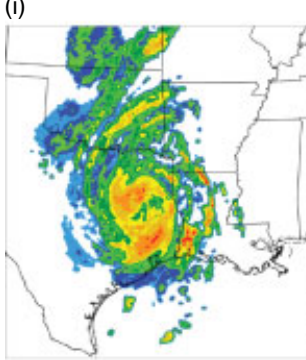

$(\mathrm{m})$

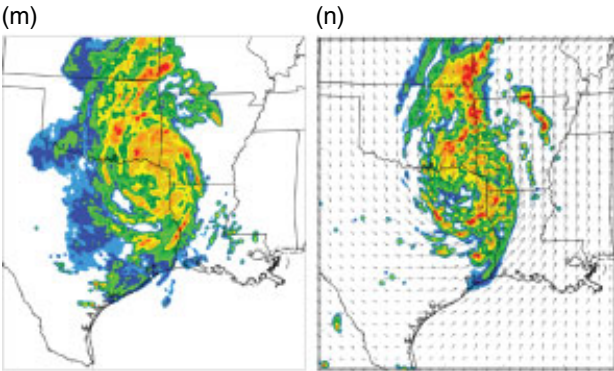

(b)
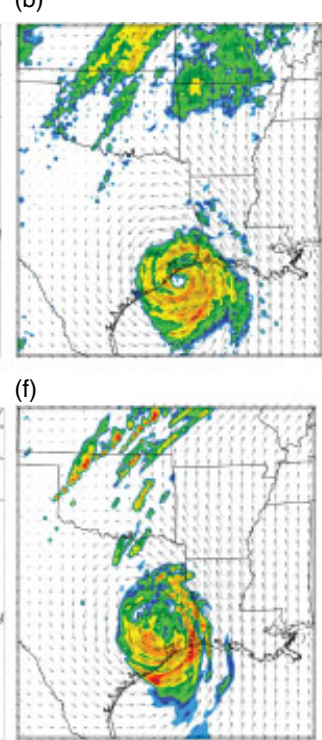

(j)
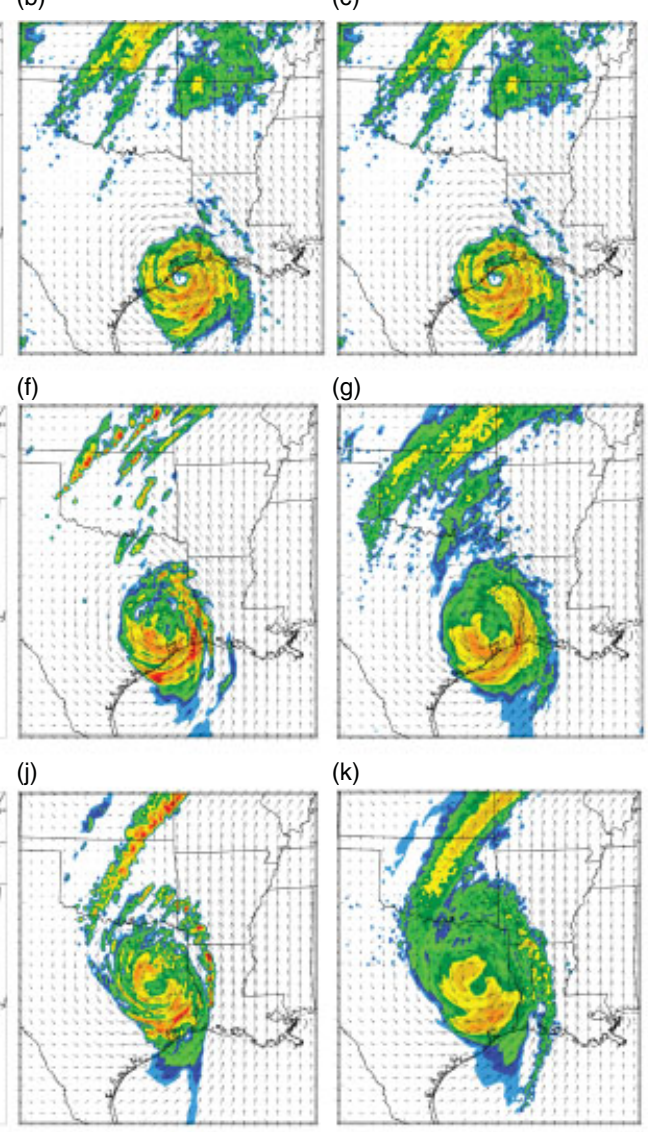

(d)
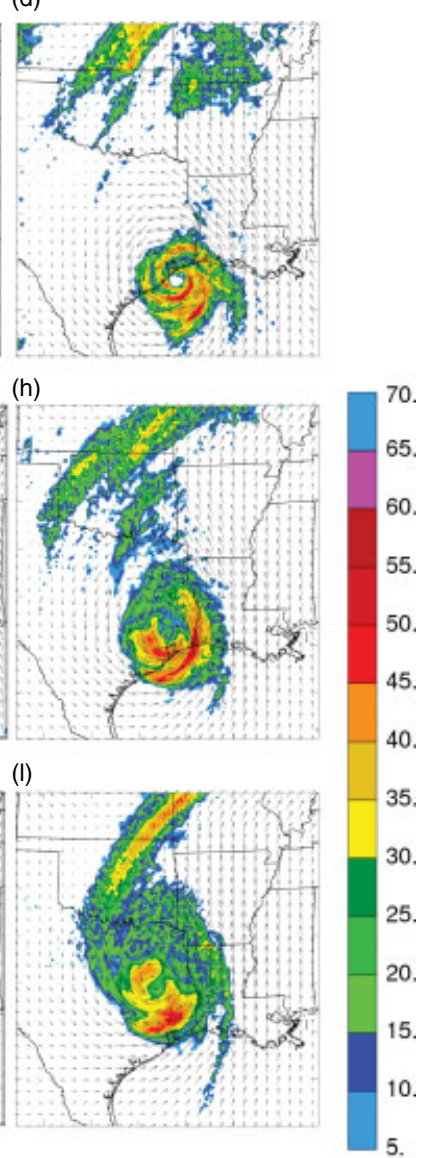

(o)

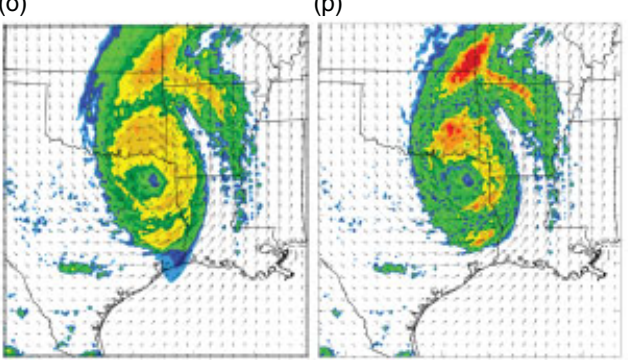

Figure 18. Composite reflectivity (colour shaded) and wind vectors at $3 \mathrm{~km}$ height analyzed and predicted by the deterministic forecast ((b), (f), (j) and $(\mathrm{n}))$, the ensemble mean forecast ((c), (g), (k) and (o)), and probability matched ensemble mean forecast ((d), (h), (l) and (p)), as compared with ((a), (e), (i) and (m)) corresponding observations. The times shown are 0600, 1200, 1800 UTC 13 September and 0000 UTC 14 September 2008 for the four rows, respectively. 


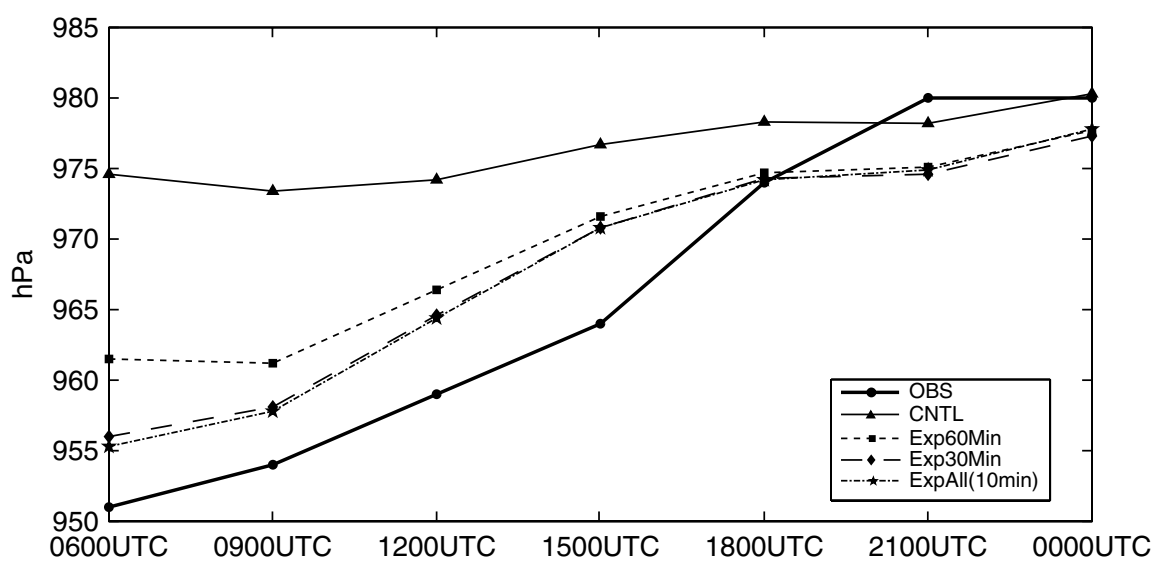

Figure 19. The predicted minimum sea-level pressures of CNTL, Exp30Min, Exp60Min and ExpAll (also Exp10Min) from 0600 UTC 13 September to 0000 UTC 14 September 2008, compared with the best track.

ensemble mean has the best ETS for all four thresholds except for $120 \mathrm{~mm}$, where the deterministic forecast scores slightly better than PM. The simulated reflectivity after PM maintains the widespread weak rainfall regions outside the vortex while it strengthens precipitation inside the hurricane (Figure 18). For example, the PM-processed $18 \mathrm{~h}$ forecast reflectivity (Figure $18(\mathrm{p})$ ) appears the closest to the observation (Figure $18(\mathrm{~m})$ ). These results show the value of an ensemble for hurricane precipitation forecasting, especially after suitable processing, while EnKF provides a potentially optimal way for generating initial condition perturbations that correctly represent analysis uncertainty.

\section{Sensitivity experiments}

\subsection{Sensitivity to assimilation interval}

To examine the sensitivity of the hurricane forecast to radar DA frequency, $Z$ and $V_{\mathrm{r}}$ data are assimilated at 30 and $60 \mathrm{~min}$ intervals in Exp30Min and Exp60Min. These experiments are otherwise the same as ExpAll. Because of the use of 10 min intervals, ExpAll is also referred to as Exp10Min in this section (Table 1).

Figure 19 shows that the $60 \mathrm{~min}$ interval is not enough to build up a vortex whose MSLP is close to that of the $10 \mathrm{~min}$ case, while the result with $30 \mathrm{~min}$ intervals is very close. The forecast in the 10 min interval case (Exp10Min) is slightly better in the first $3 \mathrm{~h}$, then becomes very close to that of Exp30Min. The track forecasts of all three experiments are similar, with mean track errors of $14 \mathrm{~km}, 12 \mathrm{~km}$ and $14 \mathrm{~km}$ for ExpAll (Exp10Min), Exp30Min and Exp60Min, respectively. The ETSs of the three cases are similar, with the ETSs of $18 \mathrm{~h}$ accumulated precipitation being $0.50,0.50$ and 0.47 for ExpAll (Exp10Min), Exp30Min and Exp60Min at the $30 \mathrm{~mm}$ threshold, and $0.20,0.20$ and 0.21 at the $120 \mathrm{~mm}$ threshold, respectively. The results suggest that the assimilation of radar data over a $2 \mathrm{~h}$ period at $30 \mathrm{~min}$ intervals may be enough, in this case at least.

\subsection{Sensitivity to single radar assimilation}

To investigate how effective data from a single Doppler radar can be, the observations from single KHGX or KLCH radar are assimilated separately in ExpKHGX and ExpKLCH (Table 1). These experiments are otherwise the same as ExpAll. The analyzed horizontal wind fields at 0600 UTC at $1 \mathrm{~km}$ height are plotted in Figure 20. In ExpKHGX (Figure 20(b)), the analyzed $1 \mathrm{~km}$ wind speed maximum is larger than in ExpKLCH (Figure 20(c)), with the region of $48 \mathrm{~m} \mathrm{~s}^{-1}$ or greater winds being much wider. Such differences may be due to the fact that the inner core of Ike is closer to KHGX than KLCH when it approaches the land. When the observations from both radars are assimilated in ExpAll (Figure 20(a)), the area of wind speed exceeding $48 \mathrm{~m} \mathrm{~s}^{-1}$ is larger than in ExpKLCH but still smaller than in ExpKHGX. On the other hand, the maximum wind at $1 \mathrm{~km}$ height is $57.9 \mathrm{~m} \mathrm{~s}^{-1}$ in ExpAll, slightly stronger than the $56.2 \mathrm{~m} \mathrm{~s}^{-1}$ in ExpKHGX and much stronger than the $51.7 \mathrm{~m} \mathrm{~s}^{-1}$ in ExpKLCH.

Among ExpAll, ExpKHGX and ExpKLCH, experiment ExpKHGX produced the strongest analyzed vortex at 0600 UTC, in terms of MSLP (Figure 21(a)). The intensity differences from the best track are 2, 11 and $4 \mathrm{hPa}$ for ExpKHGX, ExpKLCH and ExpAll, respectively. Throughout the forecast hours, ExpKHGX maintained the strongest vortex among the three. ExpKLCH produced the weakest vortex analysis and forecast, but still much stronger than in CNTL.

The track forecast of ExpKLCH is closer to the best track from 1200 to 1800 UTC with a more eastward path than ExpKHGX and ExpAll (Figure 21(b)). The track error of ExpKLCH is always the smallest or the second smallest during the $18 \mathrm{~h}$ of forecast. The mean track errors for ExpKHGX, ExpKLCH and ExpAll are 17, 9 and $14 \mathrm{~km}$, respectively.

It appears that $\mathrm{KLCH}$ has more positive contributions to the track forecast than KHGX, while KHGX has a larger positive impact on the intensity forecast than $\mathrm{KLCH}$. As in some cases the track and intensity forecasts of a hurricane tend to be more closely linked to the asymmetric and axisymmetric parts of the circulation, respectively (Emanuel, 1999; Fovell et al., 2010), it is possible that KLCH has a more positive impact on the asymmetric structure while KHGX exerts a more positive influence on the axisymmetric part of circulation. Without a reliable proof, this remains a hypothesis. In this case, assimilating both radars gives better intensity forecasts than assimilating $\mathrm{KLCH}$ alone and better track forecasts than assimilating KHGX alone. Considering that the assimilation of radial velocity data from more than one radar with different viewing angles generally improves the analysis of wind fields, the use of data from multiple Doppler radars is preferred, but the assimilation of data 


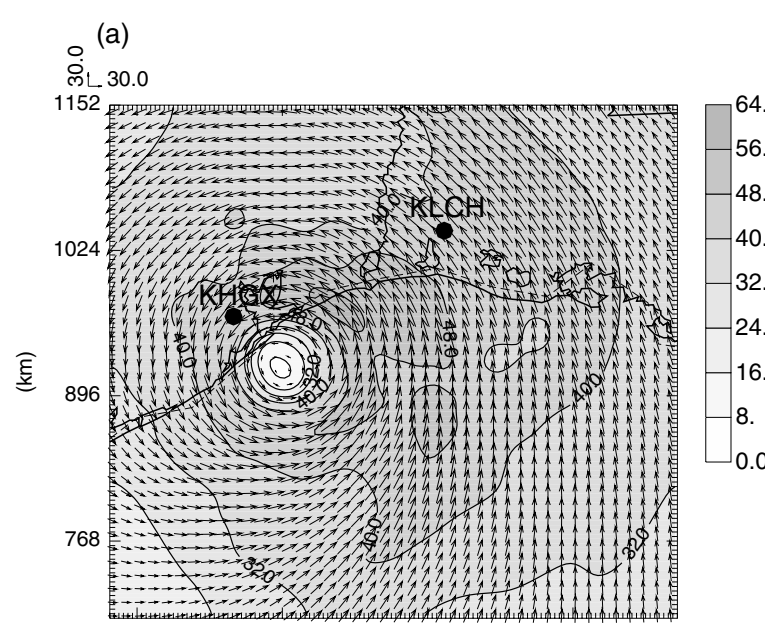

(b)
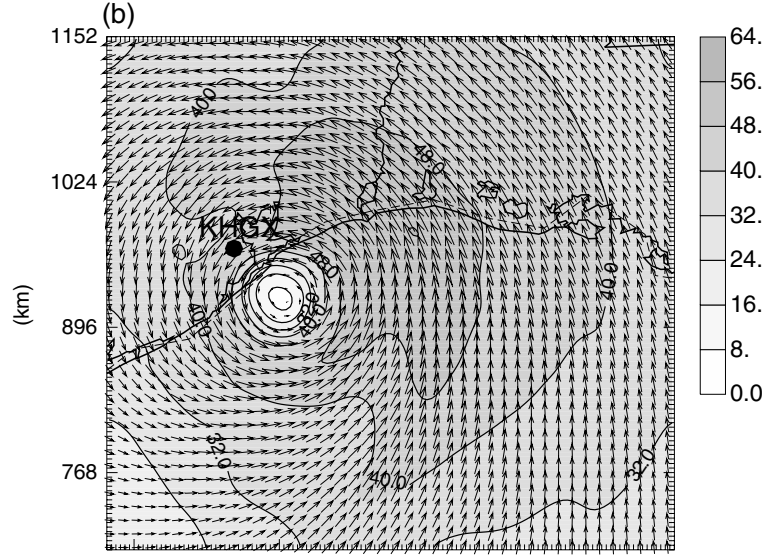

(c)

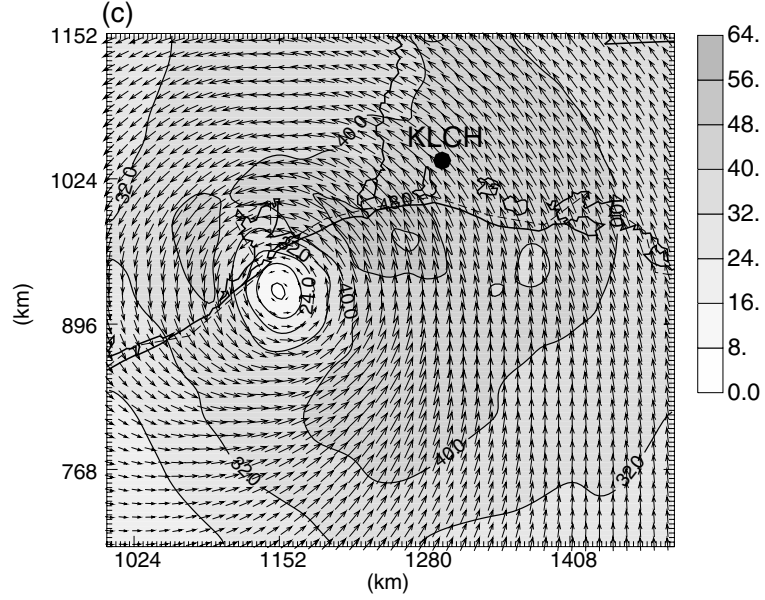

Figure 20. The analyzed horizontal wind speed (contours) vectors at $z=$ $1 \mathrm{~km}$ for (a) ExpAll, (b) ExpKHGX and (c) ExpKLCH at 0600 UTC 13 September 2008.

from a single radar can also be very beneficial; such results also indicate the power of the EnKF DA method.

\section{Summary and conclusions}

The impact of radar data assimilation on the analysis and forecasting of Hurricane Ike (2008), in terms of intensity, track and precipitation, is investigated with the cloudresolving ARPS model and the ARPS EnKF DA system. Radial velocity ( $\mathrm{Vr}$ ) and/or reflectivity $(\mathrm{Z})$ observations from up to two coastal Doppler radars are assimilated within a $2 \mathrm{~h}$ period shortly before Ike's landfall. The DA intervals of 10 , 30 and $60 \mathrm{~min}$ are examined, as is the assimilation of data from each radar alone. The results of DA experiments are compared to a forecast starting from the NCEP GFS analysis valid at the end of the DA window. The structures of the analyzed hurricane vortices from different experiments are compared, and ETSs for forecast precipitation are calculated. Ensemble forecasts from the EnKF analyses are produced, and their forecasts and derived products are compared with deterministic forecasts and observations. This is the first attempt to assimilate radar reflectivity directly for a hurricane (or tropical cyclone) using EnKF, and also the first time that the precipitation forecasts resulting from EnKF analyses are directly verified against high-resolution observations for a hurricane.

With prior multiplicative and posterior additive covariance inflations, the ensemble spread is reasonably well maintained in our EnKF DA system and large impacts from the radar observations on the analyzed wind and microphysical fields are obtained when measured by observation innovations. This is true even though the filter is probably underdispersive at the vortex scale, as indicated by the small spread in MSLP. The assimilation of radar radial velocity data in the first one or two cycles clearly strengthens the hurricane vortex found in the GFS-based background.

In our study, the assimilation of radar observations is also found to improve the structure, intensity, track and precipitation forecasts of Ike over the forecast initialized with the operational GFS analysis directly. Assimilating $V_{\mathrm{r}}$ leads to a much greater improvement in intensity than assimilating $Z$. For track forecast, $V_{\mathrm{r}}$ alone produces a slightly better forecast than $Z$ alone. Assimilating $Z$ alone results in a precipitation forecast improvement that lasted longer than using $V_{\mathrm{r}}$ alone. Assimilating both $V_{\mathrm{r}}$ and $Z$ has similar results as assimilating $V_{\mathrm{r}}$ alone, indicating a dominant role of $V_{\mathrm{r}}$ data when analyzed using EnKF for hurricanes. In our study, reflectivity is used to update pressure and microphysical variables only due to the negative impact found on the analysis when updating other variables. The covariance structure between the reflectivity and those fields might not have been reliable enough, especially during the earlier cycles, to allow for direct positive impacts on the other variables. This is an aspect that will require further investigation.

The similarity in the track and intensity forecasts when assimilating radial wind alone and assimilating both radial wind and reflectivity is also observed by $\mathrm{Pu}$ et al. (2009) in their airborne radar DA study using WRF 3D-Var. They observed small impacts on hurricane intensity and track forecasting from reflectivity data. They attributed the smaller improvement on the intensity forecast partly to a small impact on their track forecast. They also attributed the use of warm rain microphysics in their study as a possible reason. Zhao and Jin (2008) also found a marginal or negligible impact on the intensity analysis and forecasting from assimilating reflectivity alone with the US Navy's 3D-Var system and mesoscale model. Zhao and Xue (2009) noticed, however, significant improvements from reflectivity assimilation in Ike intensity forecasts when using the ARPS 3D-Var combined with a complex cloud analysis package including an ice-microphysics scheme. The adjustment to the moisture field in the complex cloud analysis procedure was found to be the most important to the intensity analysis; without the moisture adjustment, the reflectivity data had very little impact in their study (Zhao and Xue, 2009). 

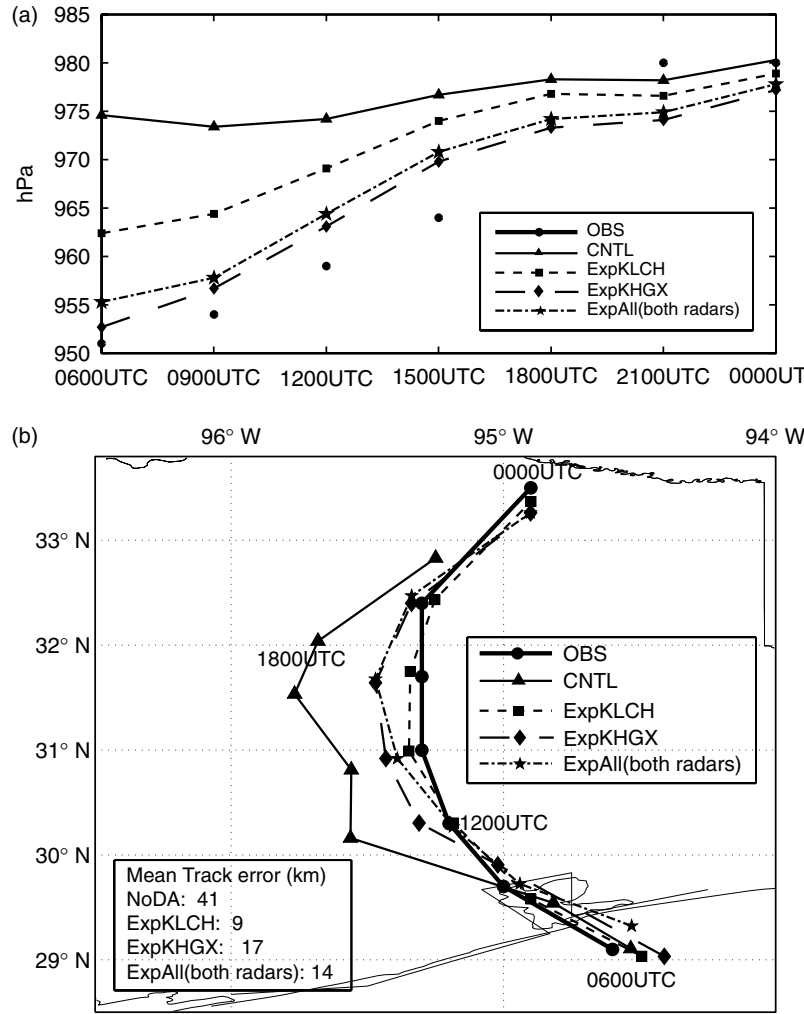

Figure 21. (a) The predicted minimum sea-level pressures and (b) the forecasted track of CNTL, ExpKHGX, ExpKLCH and ExpAll from 0600 UTC 13 September to 0000 UTC 14 September 2008, compared with the best track.

In our experiments with EnKF, which use the same forecast model as Zhao and Xue (2009), there is a solid improvement in track forecast assimilating reflectivity data alone (as much as assimilating both $Z$ and $V_{\mathrm{r}}$ ) but only a moderate improvement in intensity forecast. Because of the close involvement with the model microphysics and uncertainties with the reflectivity observation operators (e.g. Jung et al., 2010), among other things, the assimilation of reflectivity data is a more challenging problem than assimilating radial velocity. As a result, there are few studies on the impact of radar reflectivity observations in the context of hurricane forecasts, and more studies are clearly needed to investigate this topic in more depth.

Ensemble forecasts starting from our EnKF analyses show some uncertainty growth in track, but not much growth in intensity. The latter is at least partly due to the weakening of the hurricane itself during the forecast period. The simple ensemble mean precipitation forecast is found to outperform the deterministic forecast for stratiform precipitation in terms of ETSs. For high precipitation thresholds, the ensemble mean is worse. The probability matching technique for ensemble post-processing improves the ETSs at heavy rainfall thresholds. Overall, the ensemblederived probability-matched ETSs are the highest or close to the highest at all the thresholds evaluated.

The experiment with 30 min assimilation intervals shows similar results as the $10 \mathrm{~min}$ cycles, while assimilating radar data at $60 \mathrm{~min}$ intervals fails to obtain a strong vortex at the end of the $2 \mathrm{~h}$ assimilation window. The experiment with both coastal radars has a better track forecast than using the Houston-Galveston, Texas (KHGX) radar alone and a better intensity forecast than using the Lake Charles, Louisiana (KLCH) radar alone; these results indicate advantages of using data from multiple Doppler radars. The hurricane forecast assimilating data from a single Doppler radar is also much improved over that without radar data.

Radar data observe the convective and sub-vortex scales but their impact on hurricane forecast is necessarily not limited to a short period of time, especially when the vortexscale structure and intensity are improved by the data assimilation (Zhang et al., 2011). More research efforts are needed to derive maximum benefit from Doppler radar observations, including reflectivity data, in the future. In addition, other observational sources such as surface observations, soundings, profilers and satellite observations are expected to help improve the larger scale environment and improve the forecast at longer ranges. Combining all available sources of data should be the direction of future efforts. We do realize that the results obtained in this study can be case or data assimilation system dependent. Some related discussions have been provided, and other published studies are referred to. Future research should test such impacts for many cases to obtain statistically robust results and our effort in this article represents a step in that direction.

\section{Acknowledgements}

This article is mostly based on the PhD research of the first author. The authors thank Dr Kun Zhao and Ningzhu Du for their assistance and helpful discussions. Jordan Schleif is thanked for proofreading the manuscript. This work was primarily supported by NSF grant OCI-0905040, DOD ONR grants N00014-10-1-0133 and N00014-10-1-0775, and NOAA grant NA08OAR4320904. The second author was also supported by NSF grants EEC-0313747, AGS-0608168, AGS-0738370, AGS-0750790, AGS-0802888, AGS-0941491 and AGS-1046171. Computations were performed at the Pittsburgh Supercomputing Center (PSC), the National Institute of Computational Sciences (NICS) at the University of Tennessee and the Texas Advanced Computing Center (TACC) at the University of Texas at Austin.

\section{References}

Aksoy A, Dowell DC, Snyder C. 2009. A multicase comparative assessment of the ensemble Kalman filter for assimilation of radar observations. Part I: Storm-scale analyses. Mon. Weather Rev. 137: 1805-1824.

Anthes RA. 1982. Tropical Cyclones, Their Evolution, Structure and Effect. Monograph, American Meteorological Society: 208 pp.

Brewster K, Hu M, Xue M, Gao J. 2005. Efficient assimilation of radar data at high resolution for short-range numerical weather prediction. WWRP International Symposium on Nowcasting and Very Short range Forecasting, CDROM 3.06

Businger JA, Wyngaard JC, Izumi Y, Bradley EF. 1971. Flux-profile relationships in the atmospheric surface layer. J. Atmos. Sci. 28: $181-189$.

Cangialosi JP, Franklin JL. 2011. 2010 National Hurricane Center Forecast Verification Report; 77 pp.

Chan JCL, Williams RT. 1987. Analytical and numerical studies of the beta-effect in tropical cyclone motion. Part I: Zero mean flow. J. Atmos. Sci. 44: 1257-1265.

Chen SS, Price JF, Zhao W, Donelana MA, Walshc EJ. 2007. The CBLAST-Hurricane program and the next-generation fully coupled atmosphere-wave-ocean models for hurricane research and prediction. Bull. Am. Meteorol. Soc. 88: 311-317.

Clark AJ, William A, Gallus J, Xue M, Kong F. 2009. A comparison of precipitation forecast skill between small convection-permitting and large convection-parameterizing ensembles. Weather Forecast. 24: $1121-1140$. 
Davis C, Bosart LF. 2002. Numerical simulations of the genesis of hurricane Diana (1984). Part II: Sensitivity of track and intensity prediction. Mon. Weather Rev. 130: 1100-1124.

Davis C, Wang W, Chen SS, Chen Y, Corbosiero K, DeMaria M, Dudhia J, Holland G, Klemp J, Michalakes J, Reeves H, Rotunno R, Snyder C, Xiao Q. 2008. Prediction of landfalling hurricanes with the advanced hurricane WRF model. Mon. Weather Rev. 136: 1990-2005.

Deardorff JW. 1972. Parameterization of the planetary boundary layer for use in general circulation models. Mon. Weather Rev. 100: 93-106.

Deardorff JW. 1980. Stratocumulus-capped mixed layers derived from a three-dimensional model. Bound. Layer Meteorol. 18: 495-527.

Donelan MA, Dobson FW, Smith SD, Anderson RJ. 1993. On the dependence of sea surface roughness on wave development. J. Phys. Oceanogr. 23: 2143-2149.

Donelan MA, Haus BK, Reul N, Plant WJ, Stiassnie M, Graber HC, Brown OB, Saltzman ES. 2004. On the limiting aerodynamic roughness of the ocean in very strong winds. Geophys. Res. Lett. 31: L18306.

Dowell D, Zhang F, Wicker LJ, Snyder C, Crook NA. 2004. Wind and temperature retrievals in the 17 May 1981 Arcadia, Oklahoma supercell: Ensemble Kalman filter experiments. Mon. Weather Rev. 132: 1982-2005

Dowell DC, Wicker LJ. 2009. Additive noise for storm-scale ensemble data assimilation. J. Atmos. Ocean. Tech. 26: 911-927.

Dowell DC, Wicker LJ, Snyder C. 2011. Ensemble Kalman filter assimilation of radar observations of the 8 May 2003 Oklahoma City supercell: Influence of reflectivity observations on storm-scale analysis. Mon. Weather Rev. 139: 272-294.

Ebert EE. 2001. Ability of a poor man's ensemble to predict the probability and distribution of precipitation. Mon. Weather Rev. 129: 2461-2480.

Emanuel KA. 1999. Thermodynamic control of hurricane intensity. Nature 401: 665-669.

Emanuel KA. 2005. Divine Wind: The History and Science of Hurricanes. Oxford University Press; 296 pp.

Evensen G. 1994. Sequential data assimilation with a nonlinear quasigeostrophic model using Monte Carlo methods to forecast error statistics. J. Geophys. Res. 99: 10143-10162.

Fierro AO, Rogers RF, Marks FD, Nolan DS. 2009. The impact of horizontal grid spacing on the microphysical and kinematic structures of strong tropical cyclones simulated with the WRF-ARW model. Mon. Weather Rev. 137: 3717-3743.

Fiorino M, Elsberry RL. 1989. Some aspects of vortex structure related to tropical cyclone motion. J. Atmos. Sci. 46: 975-990.

Flatau M, Schubert WH, Stevens DE. 1994. The role of baroclinic processes in tropical cyclone motion: The influence of vertical tilt. J. Atmos. Sci. 51: 2589-2601.

Fovell RG, Corbosiero KL, Kuo HC. 2009. Cloud microphysics impact on hurricane track as revealed in idealized experiments. J. Atmos. Sci. 66: $1764-1778$.

Fovell RG, Corbosiero KL, Seifert A, Liou K-N. 2010. Impact of cloudradiative processes on hurricane track. Geoph. Res. Lett. 37: L07808, DOI:10.1029/2010GL042691.

Hamill TM, Whitaker JS, Fiorino M, Benjamin SG. 2011. Global ensemble predictions of 2009's tropical cyclones initialized with an ensemble kalman filter. Mon. Wea. Rev. 139: 668-688.

Houtekamer PL, Mitchell HL, Deng X. 2009. Model error representation in an operational ensemble Kalman filter. Mon. Weather Rev. 137: 2126-2143.

Houze RA, Jr., Chen SS, Smull BF, Lee W-C, Bell MM. 2007. Hurricane intensity and eyewall replacement. Science 315: 1235-1239.

Huang X-Y. 2000. Variational analysis using spatial filters. Mon. Weather Rev. 128: 2588-2600.

Jung Y, Zhang G, Xue M. 2008. Assimilation of simulated polarimetric radar data for a convective storm using ensemble Kalman filter. Part I: Observation operators for reflectivity and polarimetric variables. Mon. Weather Rev. 136: 2228-2245.

Jung Y, Xue M, Zhang G. 2010. Simulataneous estimation of microphysical parameters and atmospheric state using simulated polarimetric radar data and ensemble Kalman filter in the presence of observation operator error. Mon. Weather Rev. 138: 539-562.

Jung Y, Xue M, Tong M. 2012. Ensemble Kalman filter analyses of the 29-30 May 2004 Oklahoma tornadic thunderstorm using one- and two-moment bulk microphysics schemes. Mon. Weather Rev. 140: 1457-1475.

Khain A, Lynn B, Dudhia J. 2010. Aerosol effects on intensity of landfalling hurricanes as seen from simulations with the WRF model with spectral bin microphysics. J. Atmos. Sci. 67: 365-384.

Kong F, Xue M, Thomas KW, Gao J, Wang Y, Brewster K, Droegemeier KK, Kain J, Weiss S, Bright D, Coniglio M, Du J. 2009. A realtime storm-scale ensemble forecast system: 2009 spring experiment. 23nd Conference on Weather Analysis and Forecasting/19th
Conference on Numerical Weather Prediction, Omaha, NB. Paper 16A.3, American Meteorological Society: Boston, MA.

Krishnamurti TN, Pattnaik S, Stefanova L, Kumar TSVV, Mackey BP, O'Shay AJ, Pasch RJ. 2005. The hurricane intensity issue. Mon. Weather Rev. 133: 1886-1912.

Kwon IH, Cheong HB. 2010. Tropical cyclone initialization with a spherical high-order filter and an idealized three-dimensional bogus vortex. Mon. Weather Rev. 138: 1344-1367.

Li X, Pu Z. 2009. Sensitivity of numerical simulation of early rapid intensification of Hurricane Emily (2005) to cumulus parameterization schemes in different model horizontal resolutions. J. Meteorol. Soc. Japan. 87: 403-421.

Li Y, Wang X, Xue M. 2012. Assimilation of radar radial velocity data with the WRF ensemble-3DVAR hybrid system for the prediction of hurricane Ike (2008). Mon. Weather Rev. (in press).

Lin YL, Farley RD, Orville HD. 1983. Bulk parameterization of the snow field in a cloud model. J. Climate Appl. Meteor. 22: 1065-1089.

Lin Y, Mitchell KE. 2005. The NCEP stage II/IV hourly precipitation analyses: Development and applications. 19th Conference on Hydrology, American Meteorological Society.

Marchok T, Rogers R, Tuleya R. 2007. Validation Schemes for Tropical Cyclone Quantitative Precipitation Forecasts: Evaluation of operational models for U.S. landfalling cases. Weather Forecast. 22: $726-746$.

Marks FD, Jr. 2003. State of the science: Radar view of tropical cyclones. In: Radar and Atmospheric Science. Meteorological Monograph No. 30, American Meteorological Society; 33-73.

McFarquhar GM, Zhang H, Heymsfield G, Hood R, Dudhia J, Halverson JB, Marks F. 2006. Factors affecting the evolution of hurricane Erin (2001) and the distributions of hydrometeors: Role of microphysical processes. J. Atmos. Sci. 63: 127-150.

Oye R, Mueller C, Smith C. 1995. Software for radar data translation, visualization, editing and interpolation. 27th Conference on Radar Meteorology, American Meteorological Society; 359-361.

Powell MD, Vickery PJ, Reinhold T. 2003. Reduced drag coefficient for high wind speeds in tropical cyclones. Nature, 422:

$\mathrm{Pu} \mathrm{Z}$, Li X, Sun J. 2009. Impact of airborne Doppler radar data assimilation on the numerical simulation of intensity changes of hurricane Dennis near a landfall. J. Atmos. Sci. 66: 3351-3365.

$\mathrm{Pu}$ ZX, Braun SA. 2001. Evaluation of Bogus Vortex Techniques with Four-Dimensional Variational Data Assimilation. Mon. Weather Rev. 129: 2023-2039.

Purser RJ, Wu W-S, Parrish DF, Roberts NM. 2003. Numerical aspects of the application of recursive filters to variational statistical analysis. Part I: Spatially homogeneous and isotropic Gaussian covariances. Mon. Weather Rev. 131: 1524-1535.

Rappaport EN, Franklin JL, Avila LA, Baig SR, Beven JL, Blake ES Burr CA, Jiing J-G, Juckins CA, Knabb RD, Landsea CW, Mainelli M, Mayfield M, McAdie CJ, Pasch RJ, Sisko C, Stewart SR, Tribble AN. 2009. Advances and challenges at the National Hurricane Center. Weather Forecast. 24: 395-419.

Ren D, Xue M. 2004. A revised force-restore model for land-surface modeling. J. App. Meteorol. 43: 1768-1782.

Rogers R, Aberson S, Black M, Black P, Cione J, Dodge P, Dunion J, Gamache J, Kaplan J, Powell M, Shay N, Surgi N, Uhlhorn E. 2006. The intensity forecasting experiment: A NOAA multiyear field program for improving tropical cyclone intensity forecasts. Bull. Am. Meteorol. Soc. 87: 1523-1537.

Rogers RF, Marks FD, Marchok T. 2009. Tropical cyclone rainfall. Encyclopedia of Hydrological Sciences, Anderson MG (ed.). J. Wiley \& Sons: Chichester, UK.

Schaefer JT. 1990. The critical success index as an indicator of warning skill. Weather Forecast. 5: 570-575.

Sippel JA, Zhang F. 2010. Factors affecting the predictability of hurricane Humberto (2007). J. Atmos. Sci. 63: 1759-1778.

Snook N, Xue M, Jung J. 2011. Analysis of a tornadic meoscale convective vortex based on ensemble Kalman filter assimilation of CASA X-band and WSR-88D radar data. Mon. Weather Rev. 139: 3446-3468.

Sun W-Y, Chang C-Z. 1986. Diffusion model for a convective layer. Part I: Numerical simulation of convective boundary layer. J. Climate Appl. Meteorol. 25: 1445-1453.

Tong M, Xue M. 2005. Ensemble Kalman filter assimilation of Doppler radar data with a compressible nonhydrostatic model: OSS Experiments. Mon. Weather Rev. 133: 1789-1807.

Tong M, Xue M. 2008. Simultaneous estimation of microphysical parameters and atmospheric state with radar data and ensemble square-root Kalman filter. Part I: Sensitivity analysis and parameter identifiability. Mon. Weather Rev. 136: 1630-1648.

Wang Y. 2009. How do outer spiral rainbands affect tropical cyclone structure and intensity? J. Atmos. Sci. 66: 1250-1273. 
Weng Y, Zhang M, Zhang F. 2011. Advanced data assimilation for cloud-resolving hurricane initialization and prediction. Comp. Sci. Eng. 13(1): 40-49.

Whitaker JS, Hamill TM. 2002. Ensemble data assimilation without perturbed observations. Mon. Weather Rev. 130: 1913-1924.

Whitaker JS, Hamill TM. 2010. A simple state-dependent multiplicative inflation algorithm. The 4th Ensemble Kalman Filter Workshop.

Xiao Q, Kuo Y-H, Sun J, Lee W-C, Barker DM, Lim E. 2007. An approach of radar reflectivity data assimilation and its assessment with the inland QPF of typhoon Rusa (2002) at landfall. J. Appl. Meteorol. Climatol. 46: $14-22$.

Xiao Q, Chen L, Zhang X. 2009a. Evaluations of BDA scheme using the advanced research WRF (ARW) model. J. Appl. Meteorol. Climatol. 48: $680-689$.

Xiao Q, Zhang X, Davis C, Tuttle J, Holland G, Fitzpatrick PJ. 2009b. Experiments of hurricane initialization with airborne Doppler radar data for the advanced research hurricane WRF (AHW) model. Mon. Weather Rev. 137: 2758-2777.

Xue M, Droegemeier KK, Wong V, Shapiro A, Brewster K. 1995. ARPS Version 4.0 User's Guide; 380 pp. Available at http://www.caps.ou.edu/ARPS

Xue M, Zong J, Droegemeier KK. 1996. Parameterization of PBL turbulence in a multi-scale non-hydrostatic model. Preprint, 11th AMS Conference on Numerical Weather Prediction, Norfolk, VA. American Meteorological Society: Boston, MA; 363-365.

Xue M, Droegemeier KK, Wong V. 2000. The Advanced Regiona Prediction System (ARPS) - A multiscale nonhydrostatic atmospheric simulation and prediction tool. Part I: Model dynamics and verification. Meteorol. Atmos. Phys 75: 161-193.

Xue M, Droegemeier KK, Wong V, Shapiro A, Brewster K, Carr F, Weber D, Liu Y, Wang D. 2001. The advanced regional prediction system (ARPS) - A multi-scale nonhydrostatic atmospheric simulation and prediction tool. Part II: Model physics and applications. Meteorol. Atmos. Phys. 76: 143-166.

Xue M, Wang D-H, Gao J-D, Brewster K, Droegemeier KK. 2003. The Advanced regional prediction system (ARPS), storm-scale numerical weather prediction and data assimilation. Meteorol. Atmos. Phys 82: $139-170$.

Xue M, Tong M, Droegemeier KK. 2006. An OSSE framework based on the ensemble square-root Kalman filter for evaluating impact of data from radar networks on thunderstorm analysis and forecast. J. Atmos. Ocean Tech. 23: 46-66.

Zalesak ST. 1979. Fully multidimensional flux-corrected transport algorithms for fluids. J. Comput. Phys. 31: 335-362.

Zhang F. 2011. The future of hurricane prediction. Comp. Sci. Eng. 13: 9-12.

Zhang F, Snyder C, Sun J. 2004. Impacts of initial estimate and observations on the convective-scale data assimilation with an ensemble Kalman filter. Mon. Weather Rev. 132: 1238-1253.

Zhang F, Weng Y, Sippel JA, Meng Z, Bishop CH. 2009. Cloudresolving hurricane initialization and prediction through assimilation of Doppler radar observations with an ensemble Kalman filter. Mon. Weather Rev. 137: 2105-2125.

Zhang F, Weng Y, Gamache JF, Marks FD. 2011. Performance of convection-permitting hurricane initialization and prediction during 2008-2010 with ensemble data assimilation of inner-core airborne Doppler radar observations. Geophys. Res. Lett. 38: L15810, DOI:10.1029/2011GL048469.

Zhao K, Xue M. 2009. Assimilation of coastal Doppler radar data with the ARPS 3DVAR and cloud analysis for the prediction of Hurricane Ike (2008). Geophys. Res. Lett. 36: L12803, DOI:10.1029/2009GL 038658 .

Zhao K, Xue M, Lee W-C. 2012a. Assimilation of GBVTD-retrieved winds from single-Doppler radar for short-term forecasting of Super Typhoon Saomai (0608) at landfall. Quart. J. Roy. Meteor. Soc. 138: 1055-1071, DOI: 10.1002/qj.975.

Zhao K, Li X, Xue M, Jou BJ-D, Lee W-C. 2012b. Short-term forecasting through intermittent assimilation of data from Taiwan and Mainland China coastal radars for typhoon Meranti (2010) at landfall. J. Geophy. Res. (in press).

Zhao Q, Jin Y. 2008. High-Resolution Radar Data Assimilation For Hurricane Isabel (2003) At Landfall. Bulletin of the American Meteorological Society, 89: 1355-1372.

Zou X, Xiao Q. 2000. Studies on the Initialization and Simulation of a Mature Hurricane Using a Variational Bogus Data Assimilation Scheme. J. Atmos. Sci. 57: 836-860.

Zou X, Wu Y, Ray PS. 2010. Verification of a high-resolution model forecast using airborne Doppler radar analysis during the rapid intensification of Hurricane Guillermo. J. Appl. Meteor. Climat. 49: $807-820$. 